\title{
Augmenting Maneuverability of UUVs with Cycloidal Propellers
}

\author{
Manavendra Desai ${ }^{1}$, Ruddhi Gokhale ${ }^{1}$, Atanu Halder ${ }^{2}$, Moble Benedict ${ }^{2}$, Yin Lu \\ Young $1,3, *$ \\ ${ }^{1}$ Department of Mechanical Engineering, The University of Michigan, Ann \\ Arbor, MI (USA), ${ }^{2}$ Advanced Vertical Flight Laboratory, Texas A\&M University, \\ College Station, TX (USA), ${ }^{3}$ Department of Naval Architecture and Marine \\ Engineering, The University of Michigan, Ann Arbor, MI (USA)
}

\section{ABSTRACT}

This paper investigates a novel concept of using cycloidal propellers to augment the maneuverability of unmanned underwater vehicles. Cycloidal propellers are cross-flow propellers that utilize $360^{\circ}$ thrust vectoring to provide agile maneuvering to surface vessels like yachts, tug boats, buoy tenders and double ended ferries, but have yet to be utilized on present-day unmanned and autonomous marine vehicles. Through $360^{\circ}$ thrust vectoring, cycloidal propellers enable surface vessels to execute spot turns, and surge in forward and backward directions with equal ease. Such compact maneuvers are difficult to execute with the conventional screw propeller-rudder setup found on marine vehicles today. On unmanned and autonomous marine vehicles, cycloidal propellers can potentially enable controlled and decoupled maneuvering in all six degrees-of-freedom while overcoming disturbances like waves and currents, and extreme flow conditions like cavitation and ventilation. This is critical for marine vehicles operating at low speeds and in restricted waters. Therefore, the objective of this paper is to study the maneuvering characteristics of a UUV driven by a screw propeller and control fins only, and compare it to that of the UUV augmented with retractable cycloidal propellers. The cases considered are a turning circle maneuver, a low-speed $180^{\circ}$ turn and a low-speed heave maneuver. A six degrees-of-freedom motion prediction model that accounts for the non-linear and coupled loads on an underwater vehicle is developed and validated. Simulation results showed that compared to conventional propulsion systems, cycloidal propellers could potentially enable more swift, compact and decoupled maneuvers in unmanned marine vehicles. Integrated with robust control systems, cycloidal propellers can be explored for use in
\end{abstract}

dynamic positioning, ocean exploration, station-keeping, seakeeping and teaming of unmanned and autonomous marine vehicles.

\section{INTRODUCTION}

Unmanned Underwater Vehicles (UUVs) are widely utilized by defense, educational and commercial institutions for operations such as oceanic exploratory surveys and environment monitoring, maintenance of offshore renewable energy farms, pipeline inspections, and minesweeping (Griffiths [2002]). Using on-board data acquisition systems, UUVs help us tap into and efficiently navigate precarious waters without risking lives of human operators.

In the applications mentioned above, UUVs generally operate at low cruise speeds and in restricted waters. Adding to the challenge of low speed undersea navigation are flora, fauna, erratic site topology, and disturbances like waves and currents. Providing capabilities for swift, compact and agile maneuvering can contribute to the control, performance and function of UUVs while serving in such dynamic environments.

Most present-day underwater vehicles are propelled and steered using screw propellers and control fins (rudders and dive planes). However, in a UUV cruising at a low speed, control fins are known to have limited maneuverability (steering forces $\propto$ inflow speed $^{2}$ ). This does not auger well for operations such as inspection, imaging and station-keeping, which are carried out at low cruise speeds and require high UUV controllability for precise maneuvering. Gimbaled thrusters (for eg. Bluefin Robotic's Remus 600 UUV) and azimuthal thrusters (for eg. Forum Energy Technologies' XLE

\footnotetext{
${ }^{*}$ Corresponding author, E-mail: ylyoung @umich.edu, Tel.: +1-7346470249.
} 
Spirit $R O V$ ) are shrouded screw propellers that can be rotated over a limited conical and/or cylindrical angle to vector thrust and provide steering and attitude control. Since the thruster can be rotated only over a limited angle (not necessarily orthogonal to the UUV body axes), any steering action induces coupled motion in multiple degrees-of-freedom (DOF) and makes precise and decoupled maneuvering difficult.

Tunnel thrusters (for eg. Naval Postgraduate School's Phoenix AUV) can provide decoupled motion in multiple DOF to an underwater vehicle. A tunnel thruster comprises of a screw propeller contained in a tunnel running through the hull of the underwater vehicle. Since the screw propeller produces thrust along its axis of rotation, having multiple tunnel thrusters in orthogonal directions allows decoupled maneuvering and control, in multiple DOF, at low UUV cruise speeds. However, multiple tunnels take up internal volume, leave less space for payload and increase vehicle drag (Deter [1997]). Therefore, in general it is difficult to obtain decoupled and controlled motion of underwater vehicles with the conventional marine propulsors.

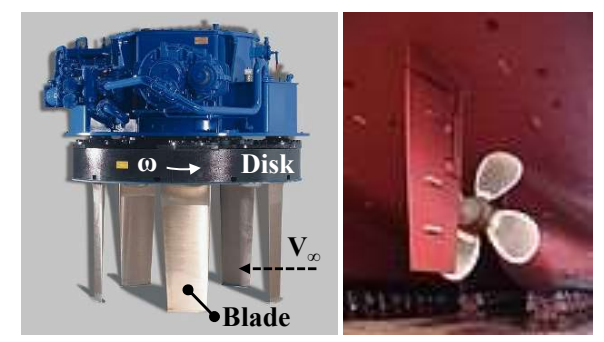

Figure 1: Voith Schneider cycloidal propeller on the left and a screw propeller-rudder combination on the right (Wikimedia [2014, 2019]). While inflow $V_{\infty}$ in a screw propeller is axial, inflow in a cycloidal propeller is perpendicular to its axis of rotation, making them cross-flow propellers. $360^{\circ}$ thrust vectoring in cycloidal propellers eliminates the need for a rudder to steer a marine vessel.

Cycloidal propellers are cross-flow propellers (Figure 1) used on marine vessels such as ships, yachts, buoy tenders, tugboats, and double-ended ferries to provide superior maneuvering and control at low vessel speeds and in restricted waters (Koschorrek et al. [2017], Jürgens et al. [2004]). On these surface vehicles, cycloidal propellers change direction of thrust (i.e. vector thrust) and provide maneuverability over $360^{\circ}$ of the surge-sway-yaw plane, eliminating the need for control fins. The compact design of cycloidal propellers make them suitable for low draught ships and boats, where maneuverability is critical. Cycloidal propellers also have a reduced tendency to cavitate (Jurgins and Heinke [2009]) and ventilate (Palm et al. [2011]) in rough seas. In offshore drilling and cable laying operations, $360^{\circ}$ thrust vectoring has enabled cycloidal propellers to outperform (the traditional) azimuthal thrusters by providing more robust dynamic positioning capabilities to marine vessels, as shown in Jürgens et al. [2012] and Jürgens et al. [2017]. However, cycloidal propellers are yet to be adopted in marine vehicles like unmanned surface vehicles and UUVs.

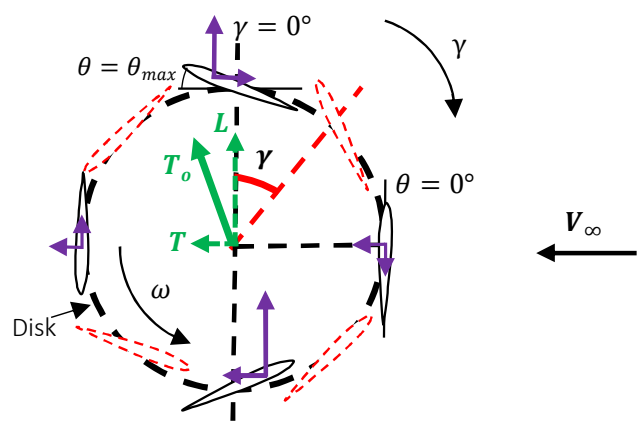

Figure 2: Sectional view of a cycloidal propeller in external flow $V_{\infty}$ producing a net force $\boldsymbol{T}_{\boldsymbol{o}}$ ( $\boldsymbol{T}$ is the thrust component and $\boldsymbol{L}$ is the lift component). While the disk rotates, the blades (simultaneously) pitch cyclically with phase $\gamma$ and a pitch angle $\theta . \quad \gamma$ is defined relative to the vertical while $\theta$ is defined relative to the tangent along the propeller disk. Starting at the top, $\gamma$ goes from $0^{\circ}$ to $360^{\circ}$ along the azimuth in the clockwise direction. In the above case, $\gamma$ is the azimuthal angle at which a blade's pitch angle $\theta$ will be maximum (i.e. $\theta=\theta_{\max }$ ). The airfoils with solid and dashed lines represent $\gamma=0^{\circ}$ and $\gamma \neq 0^{\circ}$ cases respectively.

As seen in Figure 1, cycloidal propellers comprise a rotating disk with connected blades placed perpendicular to an inflow $\boldsymbol{V}_{\infty}$. Looking at a sectional view in Figure 2, as the disk rotates with a speed $\omega$, the blades (simultaneously) pitch cyclically with a phase $\gamma$ and a pitch angle $\theta$. Cyclic pitching means that $\theta$ varies according to a particular periodic waveform (e.g. cycloidal or sinusoidal) as the blade rotates along the azimuth. In this figure, $\gamma$ is defined relative to the vertical, while $\theta$ is defined relative to the tangent along the propeller disk. $\gamma$ is equal to the azimuthal angle over the rotating disk at which the blade pitch angle $\theta$ will be maximum (i.e. $\theta=\theta_{\max }$ ). $\boldsymbol{T}_{\boldsymbol{o}}$ is the net force produced in the plane of the propeller disk. It can be decomposed into a thrust component $\boldsymbol{T}$ (along $\boldsymbol{V}_{\infty}$ ) and a lift component $\boldsymbol{L}$ (perpendicular to $\boldsymbol{V}_{\infty}$ ). $\boldsymbol{T}_{\boldsymbol{o}}$ can be varied almost steplessly in magnitude and direction over $360^{\circ}$ by altering $\omega, \gamma$ and $\theta_{\max }$, allowing swift and agile steering at low vessel speeds. The choice of $\gamma$ significantly controls the cycloidal propeller thrust direction. As a result, $360^{\circ}$ thrust vectoring makes cycloidal propellers independent of control fins and external inflow (fin steering forces $\propto$ 

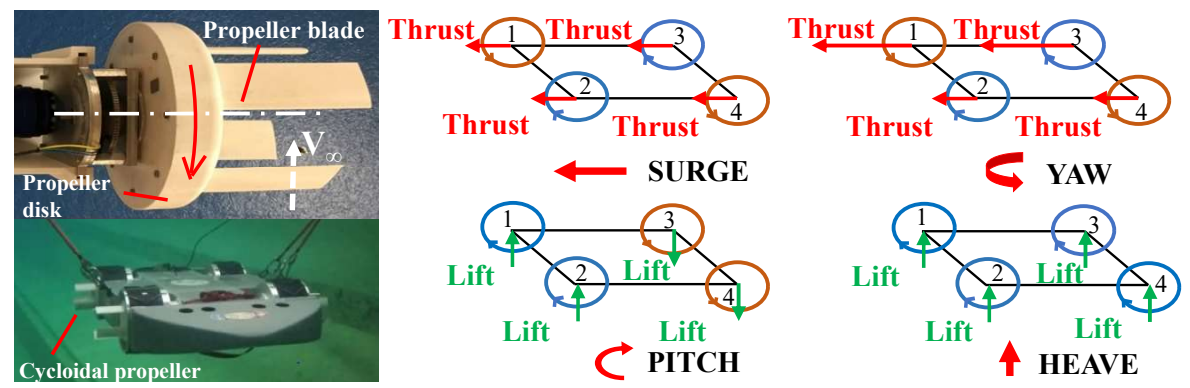

Figure 3: On the top left is a cycloidal propeller designed and manufactured in the Advanced Vertical Flight Laboratory at Texas A\&M University. It was built for a reduced scale amphibious platform, seen here on the bottom left in a towing tank test, developed as part of a collaborative project between Texas A\&M University and the University of Michigan-Ann Arbor. The amphibious platform is propelled and maneuvered solely by the four retractable cycloidal propellers. A conceptual sketch of this platform is shown on the right. The platform hull, represented by a parallelogram, is driven by four cycloidal propellers, represented by circles. The arrows on the circles give the direction of rotation of the respective cycloidal propellers. Propeller rotation speed and phase was altered to vector thrust in all four propellers, and yield differential motion in all six degrees-of-freedom. This eliminated the need for additional propulsors and control surfaces.

inflow speed ${ }^{2}$ ) for maneuvering marine vehicles.

Cycloidal propellers have already been successfully implemented in Micro-Air Vehicles (MAV) called cyclocopters (Benedict et al. [2013a], Halder et al. [2018]). Given their small size, low weight and low air resistance (primarily due to their mesh-like fuselage that allows air to flow through), the thrust produced by the cycloidal propellers is sufficient for propulsion and maneuvering in hover and forward flight. Unlike in water, cycloidal propellers in air have much lower power requirements and structural loading since the density of air is $\sim 850$ times less than that of water, and extreme flow conditions caused by cavitation and ventilation are not an issue in air (Young et al. [2017], Young [2019]).

In unmanned marine vehicles, $360^{\circ}$ thrust vectoring of cycloidal propellers can potentially provide thrust for cruise and maneuvering in all six-DOF, eliminating the need for control fins and the associated electronic systems (Figure 3). However, cycloidal propellers have traditionally been hydrodynamically less efficient than screw propellers for high speed applications, and have had a complex mechanical build. To the best of the authors' knowledge, cycloidal propellers have yet to be explored to improve the maneuverability, agility, and reduce weight and size of UUVs. Factors to be considered include mission profile, UUV size, cycloidal propeller size and configuration, as well as power and energy requirements. Such an exploration will help to advance unmanned marine vehicles (for eg. UUVs, XL[extra large]UUVs, autonomous underwater vehicles and unmanned surface vehicles) for increasingly important applications like payload delivery, equipment deployment, ocean surveying, station keeping, sea-keeping, swarming and teaming (O'Rourke [2006, 2017, 2019]).

To this end, a proof-of-concept test of a cycloidal propeller-maneuvered amphibious platform was first conducted at The Aaron Friedman Marine Hydrodynamics Laboratory at the University of Michigan, Ann Arbor (Figure 3). The amphibious platform had four retractable cycloidal propellers with independent thrust vectoring capabilities, that solely propelled and steered the platform. When driven on land, the cycloidal propellers were retracted and stowed inside the hull. The concept sketch in Figure 3 shows that such a cycloidal propeller-maneuvered platform can potentially provide thrust for cruise and steering to execute decoupled maneuvers in yaw, pitch and heave, which are difficult to attain with present day propulsors and control fins, especially at low UUV speeds and in restricted waters. Such maneuverability can be extended to all six-DOF, eliminating the need for control fins and the associated electronic systems, saving space and weight.

Therefore, the objective of the present work is to investigate maneuvering characteristics of a cycloidal propeller-augmented UUV and compare it with that of a screw propeller-control fin driven UUV. The cycloidal propeller-augmented UUV concept has cycloidal propellers in addition to a screw propeller and control fins. The cycloidal propellers will propel and maneuver the UUV at low speeds and will be used to augment the thrust and maneuverability of the screw propeller-control fin combination at higher speeds. The cycloidal propellers are envisioned to be retractable, so that they can be stowed inside the UUV hull when not 
in use and do not increase the hydrodynamic drag of the UUV.

The main contribution of this work is the development of a reduced-order, non-linear and coupled maneuvering model and MATLAB (The MathWorks, Inc) code to predict the trajectory of an underwater vehicle moving in six-DOF. This reduced order motion solver necessarily accounts for the non-linear and coupled effects of the hydrodynamic, hydrostatic and Coriolis-Centripetal forces that underwater vehicles experience. This is critical given the high density of water and the bulky make of an underwater vehicle which can result in large, coupled and destabilizing loads that severely affect vehicle maneuvering and control. Such is not the case with MAVs, where a small mesh-like fuselage flying in air generates substantially smaller resistance. In addition to being computationally cheaper than traditional CFD methods used to predict underwater vehicle trajectories, the reduced order model is also easy to couple with controller design software. This coupling can accelerate preliminary design of cycloidal propellers and the associated control systems for thrust vectoring of cycloidal propellers for precise vehicle maneuvering. The reduced order model can also form the foundation for sophisticated simulators for detailed propeller and vehicle design, and crew training. At present, the computer code, although not optimized for speed, is capable of simulation speeds at $\sim 1.1$ times the real time, on a CPU with an Intel Core i5 $2.3 \mathrm{GHz}$ processor, $8 \mathrm{~GB}$ RAM and a numerical time step of $0.01 \mathrm{~s}$.

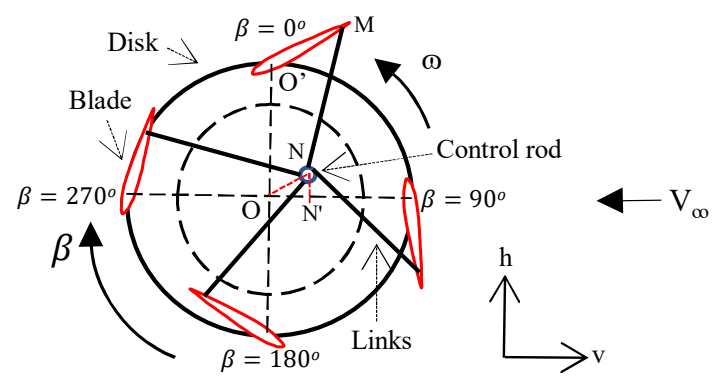

Figure 4: Simplified construction of a cycloidal propeller. The four links connect a control rod (point $N$ ) to the propeller blades at point $M$. For every rotation of the propeller disk, each blade undergoes a one-cycle oscillation in pitch, with $O N M O$ ' $O$ acting like a four bar mechanism. For a given propeller speed $\omega$, changing the length and direction of $\boldsymbol{O N}$ changes the thrust magnitude and direction respectively. Cycloidal propeller eccentricity $e=O N /(D / 2)$, where $D$ is the propeller disk diameter and $e<1$. $\omega$ and $\boldsymbol{O N}$ (length and direction) provide the three DOF for controlling cycloidal propeller thrust in magnitude and direction. In this paper, length of $O N$ is held constant.

\section{BACKGROUND}

\section{Cycloidal propellers}

Cycloidal propellers are low-pitch cross-flow propellers designed to operate at an advance coefficient $\lambda<1$ (Roesler et al. [2014]) where $\lambda=V_{\infty} / u_{p} . \quad V_{\infty}$ is the free-stream speed in the frame of the propeller, while $u_{p}$ $=\omega R$ is the propeller blade peripheral speed, and $R$ is the propeller radius.

Mechanism - Figure 4 shows a simplified sectional schematic of the cycloidal propeller mechanism. Four propeller blades are pivoted on a propeller disk and are connected to a control rod through links. As the propeller disk rotates an azimuthal angle $\beta$ about $O$, links pitch the propeller blades by an angle $\theta$ about their respective pivots $O^{\prime}$. This results in a dynamically varying blade pitch angle and a cyclic pitching motion along the azimuth. The phase angle of this cyclic pitching is $\gamma$ (Figure 2). Starting at the top, $\gamma$ and $\beta$ go from $0^{\circ}$ to $360^{\circ}$ along the azimuth in the clockwise direction. Hence, in the present paper, $\gamma$ can also be defined as the azimuthal angle $\beta$ at which the blade pitch angle $\theta$ will be maximum (i.e. $\left.\theta=\theta_{\max }\right)$. Therefore, $\gamma=0^{\circ}$ corresponds to a maximum blade pitch angle $\theta=+\theta_{\text {max }}$ at $\beta=0^{\circ}$, and $\theta=$ $0^{\circ}$ at $\beta=90^{\circ}$ and $270^{\circ}$ for each blade. For every rotation of the propeller disk, each propeller blade undergoes a one-cycle oscillation in pitch, with $O N M O O^{\prime} O$ acting like a four bar mechanism. For a given $\omega$, changing the length of $\boldsymbol{O N}$, changes $\theta_{\max }$ and the maximum obtainable thrust magnitude. Changing the direction of $O N$ changes $\gamma$ and hence the thrust direction. By varying $O N$ within the dotted circle, $360^{\circ}$ thrust vectoring is obtained. The eccentricity $e$ of the cycloidal propeller is defined as $e=$ $O N /(D / 2)$, where $D$ is the propeller disk diameter. $e<1$ as the propeller is cycloidal. The propeller speed and $O N$ (length and direction) provide the three DOF - $\omega, \theta_{\max }$ and $\gamma$ - to controlling cycloidal propeller thrust in magnitude and direction. As shown below, blade pitch angle $\theta$ is associated with the propeller blade azimuthal position $\beta$ according to Eq. (1) (Roesler et al. [2016]),

$$
\theta=\theta_{\max } \tan ^{-1}\left(\frac{e \sin \beta}{1+e \cos \beta}\right)
$$

$\theta_{\max }$ is the the blade pitch amplitude while $e(<1)$ is the eccentricity of the cycloidal propeller. 

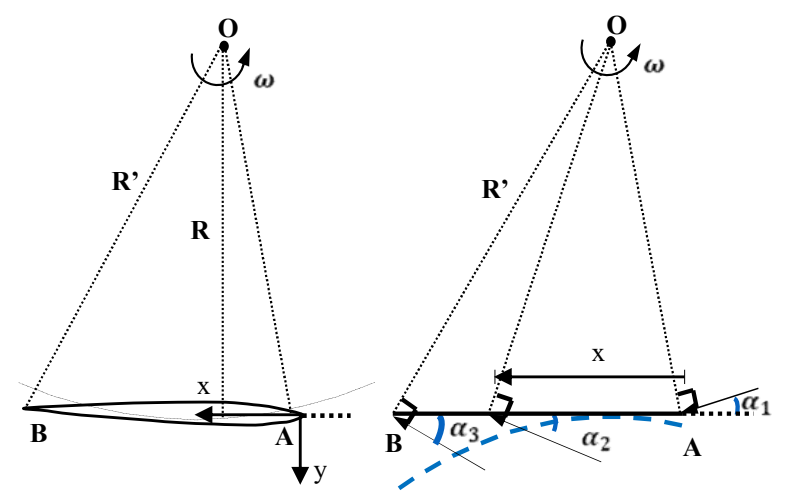

Figure 5: A cycloidal propeller blade rotating about the propeller disk axis $O$ along a curvature of radius R, with angular velocity $\omega$. The angle of attack $\alpha$ varies along the chord due to a changing radius of curvature and curvilinear velocity $(=\omega R)$. This induces a virtual camber in an otherwise symmetrical foil. This virtual camber is shown by the dashed curved line on the right.

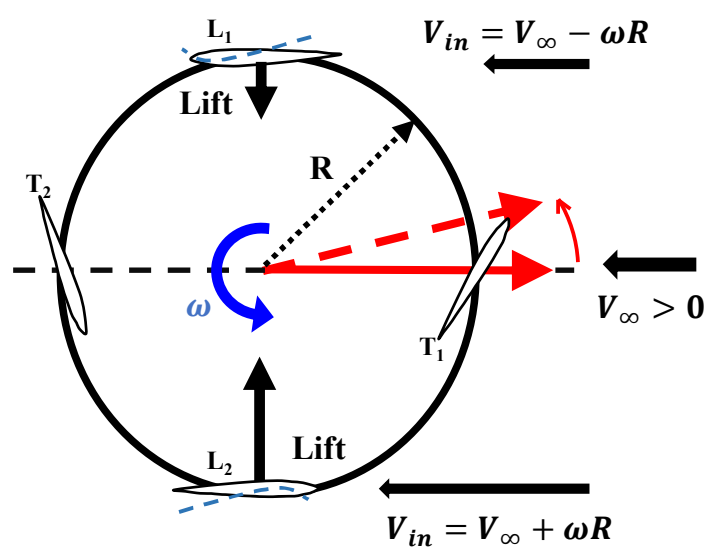

Figure 6: A non-zero free-stream velocity $\boldsymbol{V}_{\infty}$ leads to different horizontal inflow $\boldsymbol{V}_{\text {in }}$ at blades $\mathrm{L}_{1}$ and $\mathrm{L}_{2}$. Together with the virtual camber effect, this produces a non-zero lift force component that changes the cycloidal propeller net force $\boldsymbol{T}_{\mathbf{0}}$ direction (solid to dashed arrow). The new direction depends on the $V_{\infty} / \omega$ ratio. The propeller phase $\gamma$ should be changed so as to restore the direction of $\boldsymbol{T}_{\mathbf{0}}$. Hence, for $V_{\infty} \neq 0, \omega$ affects thrust direction and phase selection, and is hydrodynamically coupled with $\gamma$.

Virtual Camber - Figure 5 throws light on the inflow experienced by a cycloidal propeller blade as it moves along the azimuth. A symmetrical foil moving on a curvilinear path experiences a varying angle of attack along its chord. This is because when the propeller blade rotates about $O$, at a given azimuth angle $\beta$, the inflow velocity incidence angle varies gradually from $\alpha_{1}$ at the leading-edge $A$ to $\alpha_{3}$ at the trailing-edge $B$. Additionally, the (cyclically) varying blade pitch angle $\theta$ generates a pitch velocity ( $\propto$ blade angular velocity $\dot{\theta}$ ), resulting in a virtual camber. This virtual camber is a function of the distance $x$ from leading-edge, in the propeller blade coordinate system $x-y$. Since the propeller blade pitches about its pivot $O^{\prime}$ while the propeller disk rotates, the blade pitch $\theta$ and hence the inflow velocity is different at each azimuth position $\beta$ along the propeller blade orbit. As a result, the camber varies with $\beta$ and $\theta$, in addition to being a function of $x$. This gives rise to a dynamic virtual camber. Therefore, due to this dynamic virtual camber effect, a symmetrical foil moving on a curvilinear path can be modeled as a foil with a variable camber moving on a rectilinear path.
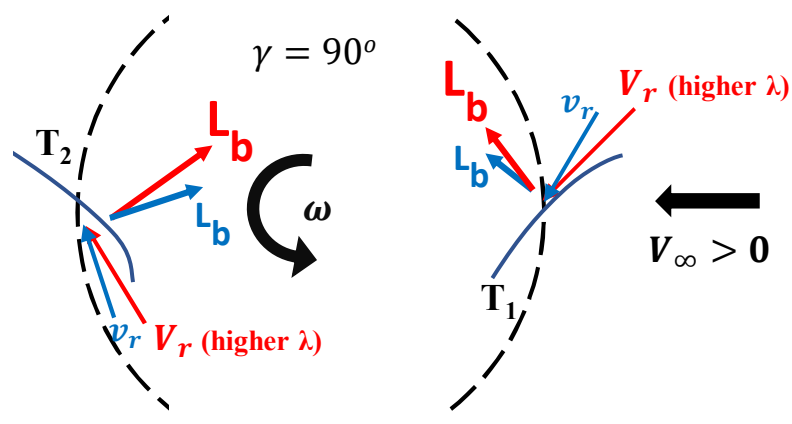

Figure 7: Effect of advance coefficient $\lambda$ on thrust producing effectiveness of a cycloidal propeller. Higher free-stream velocity $\boldsymbol{V}_{\infty}$ and advance coefficient $\lambda$ increases the flow speed $\mathrm{V}_{r}$ and reduces its incidence angle at the blades. This orients the (now larger) propeller blade forces $\boldsymbol{L}_{\boldsymbol{b}}$ more towards the vertical direction $(\beta=$ $\left.0^{\circ}\right)$, increasing the cycloidal propeller lift component $\boldsymbol{L}$ and reducing the thrust force component $\boldsymbol{T}$.

Dynamic effects - As can be seen in Figure 6, during UUV cruise at $\boldsymbol{V}_{\infty}$, there is a difference in relative inflow speed $V_{\text {in }}$ at the top $\left(\beta=0^{\circ}\right)$ and bottom $\left(\beta=180^{\circ}\right)$ propeller blades. The anti-clockwise rotation of the cycloidal propeller increases the inflow speed $V_{i n}$ at the bottom propeller blade $L_{2}$ while it reduces $V_{\text {in }}$ at the top propeller blade $L_{1}$. This results in different fluid velocities at $L_{1}$ and $L_{2}$ and hence a differential lift force. In general, blades $L_{1}$ and $L_{2}$ are responsible for lift generation while blades $T_{1}$ and $T_{2}$ are responsible for thrust generation. $T_{2}$ generates positive thrust force while $T_{1}$ usually generates a negative thrust force (Halder and Benedict [2018]). Additionally, non-zero propeller inflow $(\lambda \neq 0)$ brings out the hydrodynamic coupling between $\omega$ and $\gamma$. As stated in the section Introduction, $\gamma$ is primarily used to control the direction of the net propeller force $\boldsymbol{T}_{\mathbf{0}}$. In Figure 6, it is seen that altering $\omega$ alters $V_{\text {in }}$ and the flow dynamics at the cycloidal propeller blades. This changes 
$\boldsymbol{T}_{\mathbf{0}}$ in magnitude and direction. Hence, thrust vectoring in cycloidal propellers is affected by $\gamma$ and $\omega$, and therefore $\omega$ is hydrodynamically coupled with $\gamma$. In the case shown in Figure 6, phase $\gamma$ will have to be increased in the clockwise direction to a new phase angle $\gamma^{\prime}$ so as to vector $\boldsymbol{T}_{\mathbf{0}}$ in the horizontal direction (i.e reduce the cycloidal propeller lift component $\boldsymbol{L}$ to zero). An increase in $\boldsymbol{V}_{\infty}$ will increase the required $\gamma^{\prime}$. This can be understood in Figure 7. Here, blades $T 1$ and $T 2$ are represented by their respective virtual cambers. As $V_{\infty}$ is increased, for the same propeller rotation speed $\omega$, the advance coefficient $\lambda$ increases too. The net inflow $V_{r}$ into the propeller blades is now more influenced by $V_{\infty}$ than the circumferential component $(\omega R)$. This tilts $V_{r}$ towards the horizontal direction $\left(\beta=90^{\circ}\right)$. As a result, a higher net inflow $V_{r}$ (shown in red) is incident at a lower effective angle of attack at the blades. This orients the (now larger) propeller blade forces $\boldsymbol{L}_{\boldsymbol{b}}$ more towards the vertical direction $(\beta=$ $0^{\circ}$ ), increasing the cycloidal propeller lift component $\boldsymbol{L}$ and reducing the thrust force component $\boldsymbol{T}$. Therefore, a cycloidal propeller at a high advance coefficient $\lambda$ is less effective in producing thrust.

Based on the experimental analysis in Benedict et al. [2013a,b, 2014], a propeller blade number of four to five, and a blade pitch amplitude $\theta_{\max }$ between $25^{\circ}$ and $45^{\circ}$ favor a high power loading (ratio of propeller thrust produced and propeller power required) and thrust producing capacity for the cycloidal propeller in Figure 3. Additionally, reducing the ratio between the blade chord $c$ and propeller radius $R$ reduces the influence of the virtual camber on cycloidal propeller net force $\boldsymbol{T}_{\mathbf{0}}$. Lift force component $\boldsymbol{L}$ reduces relative to the thrust force component $\boldsymbol{T}$ and the power loading increases. A reduced-order aerodynamic model that takes into account the unsteady and virtual camber effects in a rotating cycloidal propeller has already been developed in Halder et al. [2017] and is used in the present study to simulate propeller forces for the cycloidal propeller-augmented UUV. A brief description of this model follows.

Mathematical model - Since the cycloidal propeller blades both rotate along the azimuth and pitch about their pivots, coordinate transformations are needed to get the resultant inflow velocity $\boldsymbol{V}$ in the frame of reference of the blade. $\boldsymbol{V}$ will also be a function of the position $x$ along the chord since the curvilinear flow produces a virtual camber in the foil. The geometry of a single blade can be seen in Figure 5.

$$
\boldsymbol{V}(\boldsymbol{x})=\boldsymbol{V}_{p}(\boldsymbol{x})+\boldsymbol{V}_{i}(\boldsymbol{x})+\boldsymbol{V}_{\infty}-\boldsymbol{V}_{b}
$$

Here, $\boldsymbol{V}(\boldsymbol{x})$ is the resultant inflow velocity in the propeller blade frame of reference, $V_{p}(x)$ is the linear velocity induced due to the pitching motion of the blade, $\boldsymbol{V}_{i}(\boldsymbol{x})$ is the velocity induced by an upstream propeller blade due to thrust production, and $V_{b}(x)$ is the velocity induced in propeller blade frame of reference due to curvilinear flow. The final velocity $\boldsymbol{V}(\boldsymbol{x})$ can be decomposed into corresponding $x$ and $y$ components in the blade coordinate system as shown in Figure 5.

$$
\boldsymbol{V}(\boldsymbol{x})=-V_{x} \hat{\boldsymbol{i}}-V_{y} \hat{\boldsymbol{j}}
$$

Using this formulation, the net inflow angle of attack $\alpha(x)$ in the propeller blade coordinate system can be determined as,

$$
\alpha(x)=\tan ^{-1} \frac{V_{y}}{V_{x}}
$$

Here, $V_{y}$ and $V_{x}$ are the velocity components normal and tangential to the blade orbit of rotation. Next, the coefficient of steady-state lift is determined using a thin hydrofoil theory:

$$
C_{L, s}=C_{L \alpha} \sin \left(\alpha_{e f f}\right)+C_{L o}
$$

Here, $C_{L o}$ is the lift coefficient at $\alpha_{e f f}=0$ due to the virtual camber effect. It is calculated by conformal mapping of the rotor blade coordinate system in a curvilinear flow onto a virtually cambered rotor blade coordinate system in rectilinear flow. The detailed process has been outlined in Halder et al. [2017]. $\alpha_{e f f}$ $\left(=\alpha(x)-\alpha_{i}\left(y_{o}\right)\right)$ is the effective angle of attack in the propeller blade coordinate system. Here, $\alpha_{i}$ is the induced angle of attack due to finite blade span effects and is accounted for by the use of the non-linear lifting line theory. The induced angle of attack $\alpha_{i}$ is obtained by numerically integrating the Biot-Savart equation in Eq. (6) at five Gauss quadrature points placed over a propeller blade span $b$.

$$
\alpha_{i}\left(y_{o}\right)=\frac{1}{4 \pi V_{\infty}} \int_{-b / 2}^{b / 2} \frac{d \Gamma / d y}{y_{o}-y} d y
$$

In Eq. (6), $\Gamma$ is the circulation. $y_{o}$ is the nodal position of a quadrature point along the propeller blade span. The steady state hydrodynamic lift force generated per propeller blade is calculated using the equation $L_{b}=$ $\frac{1}{2} \rho S_{\text {blade }} C_{L, s} V^{2}$, where $\rho$ is the fluid density and $S_{\text {blade }}$ is the propeller blade planform area. Circulation $\Gamma$ is then recalculated based on most recent lift $L_{b}$. This process is carried out iteratively in a numerical code until convergence is met. Circulation for the first iteration is taken from values obtained assuming elliptical lift distribution over the propeller blade span. Cyclic blade pitching leads to unsteady effects such as leading edge vortices and wake shedding. These are solved in Halder 
and Benedict [2018] using the Theodorsen's function and the Polhamus suction analogy.

$$
C_{L, u n}=a_{0} / 2+\sum_{i=1}^{n}\left(a_{n} \cos (i \omega t)+b_{n} \sin (i \omega t)\right) C\left(i k_{f}\right)
$$

In Eq. (7) shown above, $C\left(i k_{f}\right)=F\left(i k_{f}\right)+j G\left(i k_{f}\right)$ is the Theodorsen's function corresponding to a reduced frequency $k_{i}=i k_{f}$, where $i$ varies from 1 to $n$ and $k_{f}=$ $\omega c /(2 V) . a_{n}$ and $b_{n}$ are coefficients of a Fourier series decomposition of the oscillatory lift due to wake shedding and $C_{L, u n}$ is the corresponding blade lift coefficient. $\omega$ is the first harmonic of $C_{L, u n}$, a quasi-steady lift coefficient. Upon computing the final rotor blade lift and drag coefficients, we obtain the lift and drag on one blade of the cycloidal propeller. To account for multiple blades at different azimuth positions, a modified stream tube model is used to calculate the total force generated by one cycloidal propeller. The modified double multiple stream tube model is used in Halder and Benedict [2018] to calculate the net force generated by one propeller with four rotor blades.

\section{Six-DOF maneuvering model}

A reduced-order MATLAB computer code is used to solve a non-linear and coupled six-DOF equation of motion for an underwater vehicle. The framework behind this code is explained next.

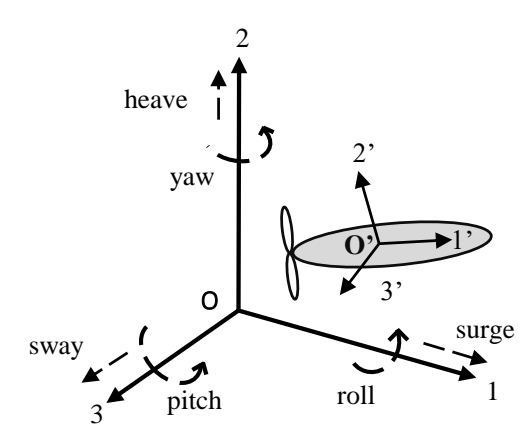

Figure 8: The coordinate systems used in the present work to study maneuvering of an unmanned underwater vehicle. $1^{\prime} 2^{\prime} 3^{\prime}$ is the body-fixed coordinate system and can be located at either the center of gravity or the center of buoyancy of the vehicle. The equation of motion is solved in this body-fixed frame of reference. 123 is the inertial coordinate system and is used to determine the vehicle trajectory.

Coordinate systems and transformations - As shown in Figure 8, two coordinate systems are utilized in studying the vehicle motion, an inertial coordinate system 123 to track vehicle trajectory and a body-fixed coordinate system 1'2'3' to calculate propulsive, self weight, hydrodynamic and hydrostatic loads on the vehicle.
Positive directions for motion in the six-DOF are shown by the dotted arrows. At each time step during simulations, 1'2'3' translates with the vehicle and assumes the same orientation as that of the vehicle, but does not rotate with it over the time step. Therefore, the vehicle will have a non-zero rotational velocity in this reference frame, bringing in Coriolis-centripetal forces and moments. 1'2'3' can be located at either the center of buoyancy or the center of gravity of the vehicle.

The equation of motion is solved for vehicle accelerations and velocities in 1'2'3'. These vectors are then oriented in 123 to solve the vehicle trajectory. This is achieved using three principle rotations about the yaw $(\psi)$, roll $(\phi)$ and pitch $\left(\theta_{v}\right)$ axes and the respective rotation matrices are provided in the Appendix. $\psi, \phi$ and $\theta_{v}$ are the Euler angles of orientation of 1'2' 3 ' in the inertial coordinate system 123 .

Mathematical model - Eq. (8) gives the six-DOF equation of motion used for the present study (Newman [2018], Hibbeler and Yap [2012], Fossen [2011]). In arriving at this formulation, it is assumed that the UUV operates at sufficiently low Froude numbers and depths so as to not produce waves at the surface (Newman [2018]). It is also assumed that the vehicle will operate in open water conditions and that currents and waves are absent.

$$
M \ddot{X}+C(\dot{X}) \dot{X}+D(\dot{X}) \dot{X}+g(\Delta)+g_{o}=\tau_{p}
$$

$M=M_{S}+M_{A}$ is the total (vehicle and fluid added) mass matrix while $C=C_{S}+C_{A}$ is the total Coriolis-centripetal force and moment matrix due to rotation of the vehicle and the fluid around it. $\boldsymbol{D}=\boldsymbol{D}_{l}+\boldsymbol{D}_{n l}$ is the combined linear $\left(D_{l}\right)$ and non-linear $\left(D_{n l}\right)$ damping matrix while $\dot{\boldsymbol{X}}=[u, v, w, p, r, q]^{T}$ is the total velocity vector of the vehicle in $1^{\prime} 2^{\prime} 3$ '. In the present work, $D$ is purely viscous and has no contributions from surface effects like radiation damping or wave drag. However, it does incorporate all the lift and drag forces and the associated moments of the underwater vessel hull and control fins. $g(\Delta)$ represents the buoyancy-driven loads, where $\Delta$ is the vehicle displacement from its hydrostatic equilibrium position. $\boldsymbol{g}_{\boldsymbol{o}}$ is the vehicle self weight load and together with $g(\Delta)$ represents the hydrostatic forces and moments. On the right is the applied propulsive forces and moments $\tau_{p}$ that act on the vehicle. Expanded forms of these matrices with the required hydrodynamic derivatives are given in Appendix B.

Numerical model - To solve for $\ddot{X}$ in time, the Coriolis-centripetal, damping, and hydrostatic loads are treated as applied forces and moments and taken to the 
right-hand side as shown in Eq. (9).

$$
\begin{array}{r}
M \ddot{X}_{n+1}=-C\left(\dot{X}_{n}\right) \dot{X}_{n}-D\left(\dot{X}_{n}\right) \dot{X}_{n}-g\left(\Delta_{n}\right) \\
-g_{o, n}+\tau_{p, n}
\end{array}
$$

$\ddot{\boldsymbol{X}}_{n+1}$ is then integrated in time using a first order explicit time integration scheme to obtain vehicle velocity $\dot{\boldsymbol{X}}_{n+1}$ and position $\boldsymbol{X}_{n+1}$ at the time step $n+1$.

\section{VALIDATION STUDIES}

To correctly study and compare the maneuvering characteristics of a screw propeller-control fin driven UUV and a cycloidal propeller-augmented UUV, the cycloidal propeller and six-DOF maneuvering models were first validated.

\section{Validation of the cycloidal propeller model}

Setup - Wind tunnel experiments were used to test a model cycloidal propeller at a free-stream speed of $3 \mathrm{~m} / \mathrm{s}$ for various propeller rotation speeds. The wind tunnel results obtained were compared with the predictions of the cycloidal propeller hydrodynamic model provided in Halder and Benedict [2018]. Table 1 lists the model propeller parameters and test parameters used for the validation experiment.

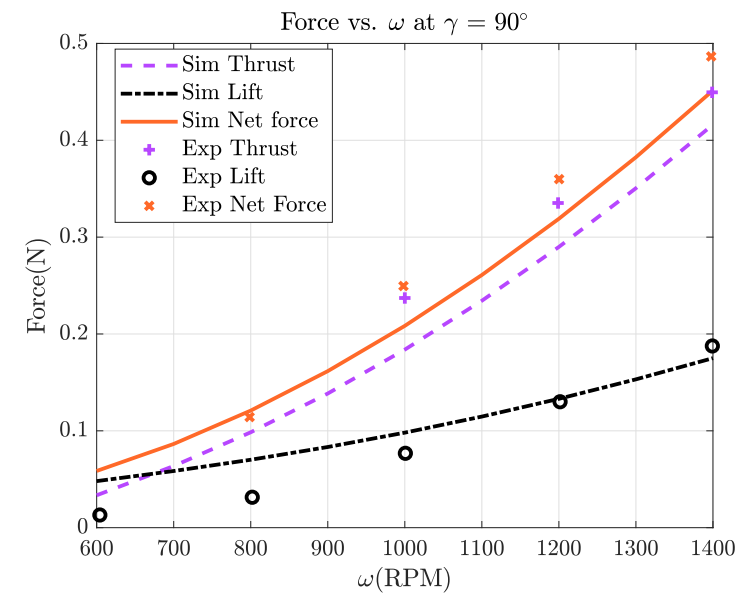

Figure 9: Lift $L$, thrust $T$ and cycloidal propeller net force $T_{0}$ as a function of propeller rotation speed $\omega$ at a phase $\gamma=90^{\circ}$ for the cycloidal propeller in Figure 3 . Data generated from the reduced order cycloidal propeller model simulation (in lines) matches well with that of the wind tunnel experiments (in markers, Halder and Benedict [2018]).
Table 1: Specifications and operating parameters for the wind tunnel test used to validate the cycloidal propeller hydrodynamic model in Halder and Benedict [2018].

\begin{tabular}{lr}
\hline Cycloidal propeller geometry and test conditions \\
\hline Number of Blades & 4 \\
Chord & $0.02 \mathrm{~m}$ \\
Radius & $0.08 \mathrm{~m}$ \\
Span & $0.16 \mathrm{~m}$ \\
Density of air & $1.22 \mathrm{~kg} / \mathrm{m}^{3}$ \\
Free-stream velocity & $3 \mathrm{~m} / \mathrm{s}$ \\
Phase angle & $90^{\circ}$ \\
Pitch amplitude angle & $45^{\circ}$ \\
\hline
\end{tabular}

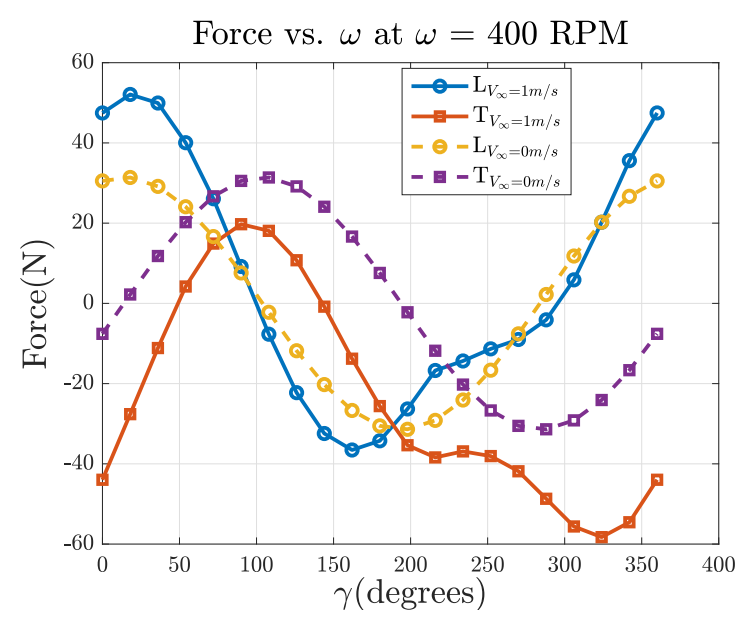

Figure 10: Simulated steady-state characteristic curves of the thrust force component $\boldsymbol{T}$ and lift force component $\boldsymbol{L}$ over $360^{\circ}$ of phase $(\gamma)$ for the cycloidal propeller in Halder and Benedict [2018], at an $\omega=400 \mathrm{rpm}$ and $V_{\infty}=[0,1] \mathrm{m} / \mathrm{s}$. At the higher free-stream speed $V_{\infty}$ of 1 $\mathrm{m} / \mathrm{s}$, the cycloidal propeller produces a higher lift force and a lower thrust force, at all $\gamma$. Hence, the thrust producing capability of a cycloidal propeller reduces with an increase in $V_{\infty}$ or advance coefficient $\lambda\left(=V_{\infty} / \omega\right)$, making it more suitable for maneuvering marine vehicles that cruise at low speeds.

Results - Figure 9 compares cycloidal propeller thrust and lift forces generated during the wind tunnel experiments, with those predicted by the hydrodynamic model. Since the virtual camber on a propeller blade varies as it moves along the azimuth $\beta$, the hydrodynamic forces too will vary with $\beta$, resulting in a time-varying lift and thrust over $360^{\circ}$ of cycloidal propeller rotation. For this purpose, time and circumferentially averaged lift and thrust forces are 
plotted in Figure 9 for comparison. As can be seen, values predicted by the code matched well with values measured during the experiments. Hence, the cycloidal propeller hydrodynamic model is validated and can be used to better understand the performance characteristics of a cycloidal propeller. For the present work, fluid density is changed to that of sea water.

Figure 10 shows the variation in propeller lift and thrust force components at $\omega=400$ rotations per minute as predicted by the cycloidal propeller hydrodynamic model. The phase $\gamma$ is varied from $0^{\circ}$ to $360^{\circ}$. As already shown in Figure 6 , thrust producing capacity of a cycloidal propeller reduces as free-stream speed $V_{\infty}$ is increased. This is evident in Figure 10. Going from a free-stream speed of $0 \mathrm{~m} / \mathrm{s}$ to $1 \mathrm{~m} / \mathrm{s}$, the thrust force component reduced while the lift force component increased in magnitude.

\section{Validation of the six-DOF maneuvering model}

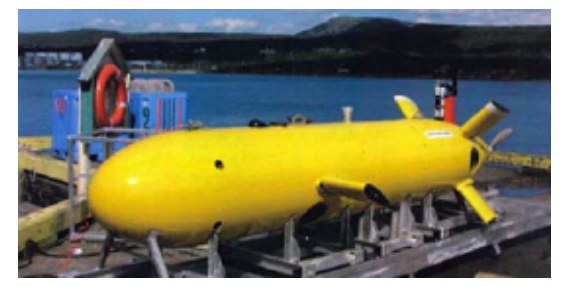

Figure 11: A 2006 Explorer AUV (Issac [2011]).
The Explorer - The Explorer (Figure 11) is a highly maneuverable and autonomous underwater vehicle (AUV) developed by International Submarine Engineering Ltd. (ISE). Its chief mission profiles include oceanic surveys, environment monitoring and pipeline inspections. The AUV is $4.5 \mathrm{~m}$ in length with a maximum diameter of $0.69 \mathrm{~m}$. It is designed to operate at depths up to $6000 \mathrm{~m}$. Propelled by a twin-blade propeller, it can reach cruise speeds between 0.5 to $2.5 \mathrm{~m} / \mathrm{s}$. On a roughly axisymmetric hull, it has a total of six control planes - two dive planes to change depth and four stern planes in an ' $\mathrm{X}$ ' configuration to provide maneuverability in roll, pitch and yaw. General vehicle specifications can be found in Table 2.

Being autonomous, the Explorer had an on-board controller and associated sensors providing data such as speed, location, trajectory, orientation, and depth. Acceleration-deceleration tests, zig-zag tests, turning circle maneuvers, and helical maneuvers were conducted as part of a research study, results of which can be found in the doctoral thesis Issac [2011].The control plane deflections and propeller rotation speed were recorded. During these sea-trial tests, a reference path and target speed was provided to the on-board controller through a mission planning and supervision software FleetManager (Fleetmanager [2020]). The sea-trial data in Issac [2011] served as a good reference to test and refine the six-DOF model and code developed in the present work.

Screw propeller and Control plane model - The Explorer $\overline{\mathrm{AUV}}$ is propelled by a dual blade screw propeller and maneuvered by six control planes. The on-board data acquisition system time-logged the propeller rotational speed and the AUV cruise speed. The propeller thrust $T_{p}$ and reaction torque $Q_{p}$ were curve fit as a function of the AUV cruise speed in Issac [2011]. In this paper, $T_{p}$ and $Q_{p}$ act solely in direction $l^{\prime}$ in the vehicle reference frame 1'2'3' and together with the resulting moments $M_{p}=l \times T_{p}$, are incorporated as $\tau_{p}$ in Eq. (8). Here $l$ is the thrust line vector in 1 '2'3'. Manufacturer-provided performance sheets for the thrust coefficients $K_{T}$, torque coefficients $K_{Q}$ and efficiency $\eta$ over a range of advance coefficients $\lambda$ have been provided in Issac [2011]. The Taylor wake fraction and thrust deduction factor of the Explorer AUV were 0.2 and 0.1 respectively. Using this knowledge and straight-line acceleration-deceleration sea-trial results, AUV hull drag and control plane drag were plotted as a function of the AUV cruise speed. An AUV hull drag coefficient of 0.0143 was inferred. During the sea-trial tests, the Explorer AUV speed varied between $1 \mathrm{~m} / \mathrm{s}-2.5 \mathrm{~m} / \mathrm{s}$, yielding an operating Reynolds number (for UUV length and speed) of the order of $10^{5}$. 


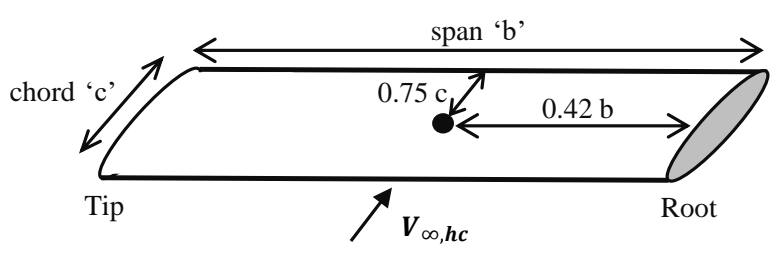

Figure 12: A control plane on the 2006 Explorer AUV (not to scale). It has a span $b$ and chord $c$ of $0.36 \mathrm{~m}$ and hence a geometric aspect ratio of one. Since the control plane is connected at its root to the UUV hull, the effective aspect ratio of the control plane is two. Hydrodynamic forces and moments produced by the control plane are calculated at the hydrodynamic center $h c$, using the flow $V_{\infty, h c}$ incident at $h c$.

The control planes are modeled as NACA 0025 hydrofoils. The control plane lift and drag forces, and moments generated are calculated at the respective hydrodynamic centers $h c$ as shown in Eq. (10) - (12) (Issac [2011]). Since the UUV hull acts like a wall at the control plane root, the control plane effective aspect ratio doubles from one to two (Figure 12).

$$
\begin{gathered}
V_{\infty, h c}=U+\boldsymbol{\Omega} \times \boldsymbol{r}_{h c} \\
\boldsymbol{F}_{f i n}=\left[\begin{array}{c}
D_{f i n} \\
L_{f i n}
\end{array}\right]=\frac{1}{2} \rho\left|\boldsymbol{V}_{\infty, h c}\right|^{2} S_{f i n}\left[\begin{array}{c}
-C_{D, f i n} \\
C_{L, f i n}
\end{array}\right] \\
M_{f i n}=r_{h c} \times F_{f i n}
\end{gathered}
$$

$\boldsymbol{V}_{\infty, h c}$ is the effective velocity incident at $h c$ due to the translational and rotational motion of the AUV. $\Omega$ is the angular velocity of rotation of the AUV in 1'2'3'. $\boldsymbol{r}_{h c}$ is the position vector of $h c$ of the control plane in the body centered frame 1'2'3'. Planform area $S_{\text {fin }}$, lift $C_{L, f i n}$ and drag $C_{D, \text { fin }}$ coefficient data for the Explorer hydrofoils are provided in Issac [2011]. Forces $\boldsymbol{F}_{f i n}$ and moments $\boldsymbol{M}_{\text {fin }}$ are transformed into the frame of the vehicle 1'2'3' and incorporated into Eq. (1) as part of the nonlinear damping matrix $\boldsymbol{D}_{n l}$ in Eq. (8). Similar calculations are made for the Explorer hull hydrodynamic forces and moments, and have been explained in detail in Issac [2011] and Evans and Nahon [2004]. Added mass coefficients, vehicle geometry and data necessary for hydrostatic load calculations are also provided in Issac [2011].

Maneuvering tests for validation - Turning Circle maneuver (TCM) and Horizontal Zig-Zag (HZZ) sea-trial tests are used to validate the six-DOF underwater vehicle trajectory prediction code. Traditionally, a TCM is used to test effectiveness of a rudder in sea-going vehicles. Such a maneuver will be of particular importance to UUVs when there will be a point of interest to be circled at length for better observation and data collection. An HZZ is traditionally used to test the turning ability of sea vehicles around obstacles, like seamounts or ship wreckage. Time-logged control plane deflections, steady-state speed and initial vehicle attitudes provided in Issac [2011] are used as inputs in the six-DOF maneuvering code. The results of our simulations are compared with those of the sea-trial tests. The TCM and HZZ sea-trial tests in Issac [2011] were performed at a depth of $3 \mathrm{~m}$ and cruise speeds of $1.5 \mathrm{~m} / \mathrm{s}$ and $1 \mathrm{~m} / \mathrm{s}$. This yielded a Froude number $=\left(\frac{\text { UUV surge speed }}{\sqrt{\text { g×UUV depth }}}\right)$ of $\sim 0.28$ and $\sim 0.18$ for the Explorer along with a depth to body diameter ratio of $\sim$ 4.4. This satisfied the assumption to ignore waves and free surface effects in the maneuvering model explained earlier (Faltinsen [2005]). In the simulations to follow, the $\mathrm{X}, \mathrm{Y}$ and $\mathrm{Z}$ axes represent the surge, heave and sway degrees-of-freedom respectively.

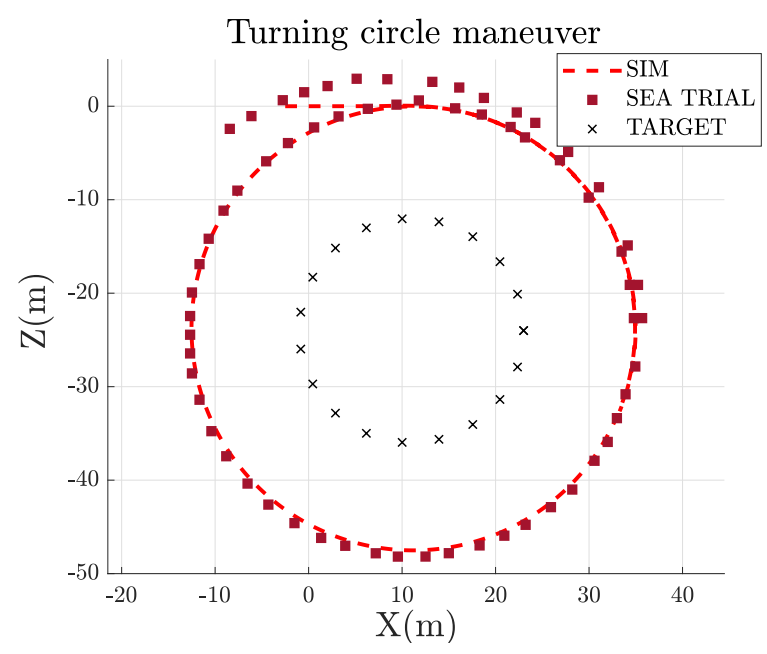

Figure 13: A comparison between sea-trial and simulated turning circle maneuver trajectories of the UUV shown in Figure 11. The sea-trial test had a turning radius of $24.2 \mathrm{~m}$. Control plane deflections chosen by the Explorer AUV control system during the steady phase of the sea-trial were averaged over time and used in this simulation, which yielded a turning radius of $23.9 \mathrm{~m}$. The target speed of $1.5 \mathrm{~m} / \mathrm{s}$ was maintained throughout the simulation. The difference in radii is attributed to a neglect of cross-currents in the sea and the use of time-averaged (and not the true instantaneous) control plane deflections.

Turning circle maneuver test - For the TCM sea-trial, the control system on-board the Explorer AUV was given the turn radius, center coordinates of the circle and turn 
radius as inputs. Figure 13 compares the Explorer AUV sea-trial trajectory with the corresponding maneuvering code prediction for a reference turning radius of $12 \mathrm{~m}$. It is seen that a larger turning radius was obtained during the sea-trial and the author of Issac [2011] suggests improper controller gains might have been the cause. This does not affect the validation exercise since the control surface deflections, chosen by the AUV control system during the sea-trial tests, are used in the simulation. While the sea-trial result had a turning radius of $24.2 \mathrm{~m}$, the maneuvering code simulates a turning radius of $23.9 \mathrm{~m}$. The target speed of $1.5 \mathrm{~m} / \mathrm{s}$ is maintained throughout the simulation. The difference in radii is attributed to the neglect of cross-currents in the sea and the use of time-averaged (and not the true instantaneous) control plane deflections.

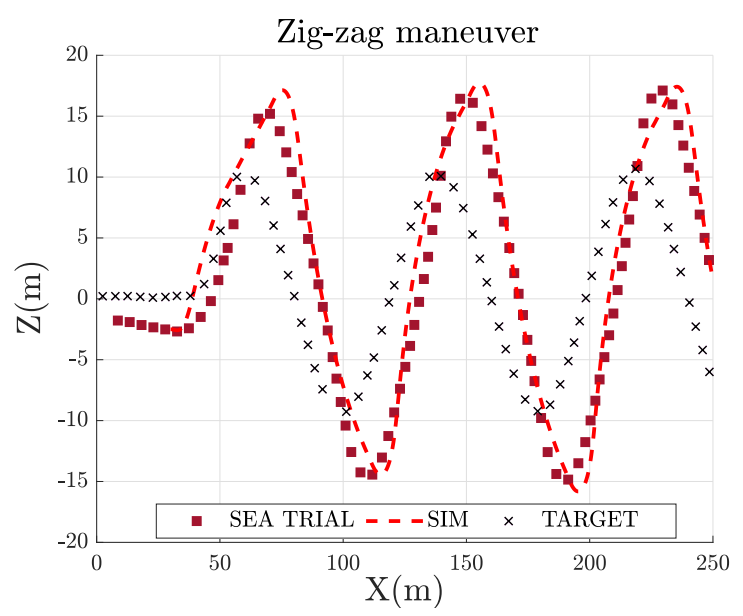

Figure 14: A comparison between sea-trial and simulated horizontal zig-zag maneuver trajectories of the UUV shown in Figure 11. Although the target sinusoidal path had a wavelength of $80 \mathrm{~m}$ and an amplitude of $10 \mathrm{~m}$, during the sea-trial the UUV overshot the target trajectory for most part of the test. The author in Issac [2011] cites improper control system gains as the probable cause. Waypoints for this sinusoidal reference trajectory were provided at its crests and troughs. Control plane deflections chosen by the Explorer AUV control system during the steady phase of the sea-trial were averaged over time and used, and the reduced order six-DOF model closely predicts the zig-zag trajectory. The difference in sinusoidal trajectories is attributed to the neglect of cross-currents in the sea and the use of time-averaged (and not the true instantaneous) control plane deflections.

Horizontal zig - zag maneuver test - For the HZZ sea-trial, crests and troughs of a reference sinusoidal path were given as waypoints to the on-board control system. During the sea-trial, the control system changed (reversed) rudder deflection only when a waypoint was crossed. This is unlike a conventional HZZ maneuver wherein the rudder angle of the marine craft is reversed once the target heading is achieved. The resulting time lag in correcting the heading caused an overshoot, as can be seen in Figure 14. Also, at the turns the controller overcompensated for a drop in speed by raising the the surge speed beyond $1.5 \mathrm{~m} / \mathrm{s}$, leading to a further increase in overshoot (Issac [2011]). Nonetheless, time-averaged deflections provided in Issac [2011] are used to simulate the HZZ maneuver. As can be seen in Figures 13 - 14, the simulated and sea-trial trajectories match well. Difference in the simulated and actual trajectories is attributed to the neglect of cross-currents in the sea and the use of time-averaged (and not the true instantaneous) control plane deflections. Since the six-DOF maneuvering model closely simulates the sea-trial tests, it is coupled with the cycloidal propeller hydrodynamic model to simulate and compare maneuvering characteristics of a screw propeller-control fin driven UUV with that of a cycloidal propeller-augmented UUV.

\section{CYCLOIDAL PROPELLER VS. SCREW PROPELLER}

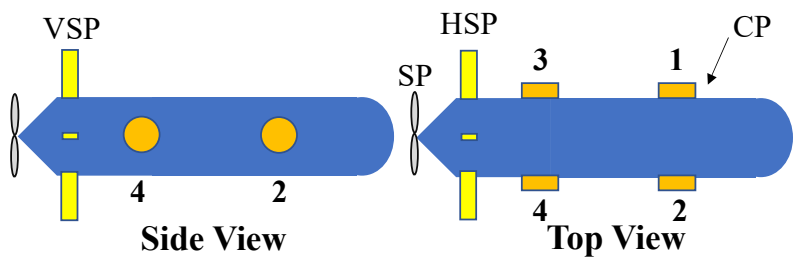

Figure 15: A simplified schematic of the cycloidal propeller-augmented Explorer AUV. The dive planes seen in Figure 11 have been removed while the hull and stern planes remain the same. HSP is a horizontal stern plane and VSP is a vertical stern plane (rudder). SP is the twin-blade screw propeller. $\mathrm{CP}$ is the cycloidal propeller.

The maneuvering characteristics of a screw propeller-control plane driven UUV, augmented with cycloidal propellers, are explored in the present work. The screw propeller and control planes remain on the augmented UUV since the cycloidal propellers will have high power requirements if made to propeller and maneuver the UUV at high cruise speeds, as already explained in the section Cycloidal propellers. The cycloidal propellers will propel and maneuver the augmented UUV at low cruise speeds, and will be used to augment the thrust and maneuverability provided by the screw propeller-control fin combination at high UUV cruise speeds. The cycloidal propellers are envisioned to be retractable, similar to the amphibious vehicle shown in the lower left hand corner of Figure 3, so that they can 
Table 3: Sea-trial and simulated turning circle maneuver results for commanded radii $R_{T, c}=12 \mathrm{~m}, 16 \mathrm{~m}, 20 \mathrm{~m}, 25 \mathrm{~m}$ and $30 \mathrm{~m}$, at $1 \mathrm{~m} / \mathrm{s}$ and $1.5 \mathrm{~m} / \mathrm{s}$, for the UUV shown in Figure 11. $u$ is the steady-state UUV speed during the sea-trial. $R_{T}$ is the turning radius executed while $\theta_{v}$ is the steady-state pitch during the turn. $r$ and $\beta_{v}$ are the steady-state yaw rate and drift angles. The subscripts MUN and SIM denote the MUN-conducted sea-trial and the present work's six-DOF maneuvering code simulation results respectively.

\begin{tabular}{cccccccccc}
\hline $\begin{array}{c}R_{T, c} \\
(\mathrm{~m})\end{array}$ & $\begin{array}{c}u \\
(\mathrm{~m} / \mathrm{s})\end{array}$ & $\begin{array}{c}R_{T, M U N} \\
(\mathrm{~m})\end{array}$ & $\begin{array}{c}R_{T, S I M} \\
(\mathrm{~m})\end{array}$ & $\begin{array}{c}\beta_{v, M U N} \\
(\mathrm{deg})\end{array}$ & $\begin{array}{c}\beta_{v, S I M} \\
(\mathrm{deg})\end{array}$ & $\begin{array}{c}r_{M U N} \\
(\mathrm{deg} / \mathrm{s})\end{array}$ & $\begin{array}{c}r_{S I M} \\
(\mathrm{deg} / \mathrm{s})\end{array}$ & $\begin{array}{c}\theta_{v, M U N} \\
(\mathrm{deg})\end{array}$ & $\begin{array}{c}\theta_{v, S I M} \\
(\mathrm{deg})\end{array}$ \\
\hline \multirow{2}{*}{16} & 1.5 & 24.2 & 23.9 & 5.6 & 5.1 & 3.5 & 3.3 & -1.6 & -1.3 \\
& 1.0 & 23.8 & 25.5 & 5.8 & 4.5 & 2.4 & 2.1 & -5.0 & -9.1 \\
\hline \multirow{2}{*}{20} & 1.5 & 21.5 & 20.1 & 2.3 & 5.9 & 3.9 & 4.0 & -1.9 & -1.8 \\
& 1.0 & 26.6 & 28.5 & 5.2 & 4.0 & 2.2 & 2.0 & -5.5 & -8.8 \\
\hline \multirow{2}{*}{25} & 1.5 & 24.7 & 24.1 & 2.0 & 5.0 & 3.5 & 3.4 & -1.9 & -0.9 \\
& 1.0 & 29.7 & 32.4 & 5.2 & 3.8 & 2.0 & 1.9 & -5.2 & -8.6 \\
\hline \multirow{2}{*}{30} & 1.5 & 28.8 & 29.3 & 1.7 & 4.2 & 3.0 & 2.8 & -1.9 & -0.5 \\
& 1.5 & 33.3 & 34.4 & 1.5 & 3.7 & 2.6 & 2.4 & -1.8 & -0.4 \\
\hline
\end{tabular}

be stowed inside the UUV hull when not in use and do not increase the hydrodynamic drag of the UUV. While conceptualizing the cycloidal propeller-augmented UUV, a few simplifying assumptions are made.

1. Four cycloidal propellers are placed symmetrically around the UUV center of buoyancy, in a horizontal plane, as shown in Figure 15.

2. There are no dive planes in the cycloidal propeller-augmented UUV. UUV depth will be altered by $360^{\circ}$ thrust vectoring of the cycloidal propellers instead.

3. In both UUVs, the center of gravity coincides with the center of buoyancy in the horizontal plane and has a vertical separation only (same as that in the 2006 Explorer AUV).

4. The UUVs are neutrally buoyant.

5. Propeller-propeller and propeller hull interaction effects have been neglected.

6. Additional inertia and buoyancy of the cycloidal propeller are assumed to be much smaller than that of the UUV and are therefore ignored.

7. Both UUVs operate at low cruise speeds in deep water and the cycloidal propeller blades are assumed to never cavitate, ventilate or bend under hydrodynamic loads.
8. Change in control plane deflections are assumed to be instantaneous, i.e, there is no time lag.

It should be remembered that the cycloidal propeller configuration used in the present work is not necessarily ideal, and will always depend on the mission profile and the application of interest.

\section{Sizing the cycloidal propellers}

Steady-state characteristic curves of the cycloidal propellers are used to select a suitable operating point to simulate the cycloidal propeller. The cycloidal propeller designed in the Advanced Vertical Flight Laboratory at Texas A\&M University (Figure 3) is used as the baseline model, dimensions of which are provided in 4 .

Table 4: The baseline geometry of the cycloidal propeller in Figure 3.

\begin{tabular}{lr}
\hline \multicolumn{2}{c}{ Baseline propeller parameters } \\
\hline Number of Blades & 4 \\
Blade profile & NACA 0012 \\
Chord & $0.05 \mathrm{~m}$ \\
Radius & $0.08 \mathrm{~m}$ \\
Span & $0.13 \mathrm{~m}$ \\
\hline
\end{tabular}


Table 5: The scaled cycloidal propeller geometry used to simulate maneuvers of the cycloidal propeller-augmented UUV.

\begin{tabular}{lr}
\hline \multicolumn{2}{c}{ Scaled propeller parameters } \\
\hline Number of Blades & 4 \\
Blade profile & NACA 0012 \\
Chord & $0.05 \mathrm{~m}$ \\
Radius & $0.18 \mathrm{~m}$ \\
Span & $0.18 \mathrm{~m}$ \\
\hline
\end{tabular}

The Explorer AUV hull had a diameter of $\sim 0.69$ $\mathrm{m}$ and control planes of span $\sim 0.36 \mathrm{~m}$. It is assumed that the cycloidal propeller can have a propeller disk with a diameter half that of the hull, and a blade span half that of the control plane. The propeller blade chord was kept the same as that in the baseline model. Dimensions of this scaled cycloidal propeller are shown in Table 5. The geometric blade aspect ratio increased to 3.5 from 2.5 and the blade chord $c$ over propeller disk radius $R$ ratio was reduced to 0.29 from 0.66. A lower $c / R$ ratio is good for thrust production and lowers cycloidal propeller power requirements (Benedict et al. [2013a]). An increased aspect ratio increases the hydrodynamic efficiency of the blades and the operational efficiency of the cycloidal propeller.

To generate the steady-state performance curves of the scaled cycloidal propeller, operating parameters are selected as follows. Propeller blade pitch amplitude $\theta_{\max }$ is set as $45^{\circ}$ for the upstream stroke and $35^{\circ}$ for the downstream stroke. This combination of angles is found to be most effective in producing thrust for the cycloidal propeller in Figure 3 (Benedict et al. [2013b]). The cycloidal propeller phase $\gamma$ is set to $96^{\circ}$. Unlike in Halder et al. [2017], the propeller blade pitch angle was chosen to vary cycloidally, and not sinusoidally, about its pitch axis as this yielded better operating efficiencies. The eccentricity $e$ in Eq. (1) is set as 0.48. The number of blades $N_{b}$ is chosen to remain as four since this provided superior power loading over the blade pitch amplitude range of $25^{\circ}$ to $45^{\circ}$ (Benedict et al. [2010]). The lift-angle of attack curve slope $C_{l_{\alpha}}$ of the propeller blades is assumed to remain unchanged and is calculated as follows (Roesler et al. [2014]),

$$
C_{l_{\alpha}}=\frac{2 \pi}{1+\left(\frac{2}{2 \times \text { Blade aspect ratio }}\right)}
$$

The blade aspect ratio used above is 3.5. Steady-state performance curves are generated for an advance coefficient $\lambda \in(0,1)$ and a cycloidal propeller inflow speed of $0.5 \mathrm{~m} / \mathrm{s}$. Although the UUV speed will not affect the performance curves of the vehicle, a low speed was chosen since the goal of the present work is to show the potential advantages of a cycloidal propeller-augmented UUV at low UUV cruise speeds. Eq. (14) - (16) define the non-dimensional time-averaged coefficients (Voith [2019]) used to generate the steady-state characteristic curves for the scaled cycloidal propeller.

$$
\begin{gathered}
K_{T}=\frac{\text { Thrust }}{\frac{1}{2} \rho d b u^{2}} \\
K_{Q}=\frac{\text { Torque }}{\frac{1}{4} \rho d b u^{2}} \\
\eta=\lambda\left(\frac{K_{T}}{K_{Q}}\right)
\end{gathered}
$$

$K_{T}, K_{Q}$ and $\eta$ are the cycloidal propeller thrust coefficient, torque coefficient and hydrodynamic efficiency respectively. Thrust and Torque are the thrust and torque produced by the propeller. $n$ is the propeller speed in rotations per second. $d$ and $b$ are the propeller diameter and blade span respectively. $\lambda=V_{\infty} /(\pi n d)$ is the advance coefficient and $V_{\infty}$ is the free-stream speed. $u_{p}=\pi n d$ is the peripheral speed of the propeller blades.

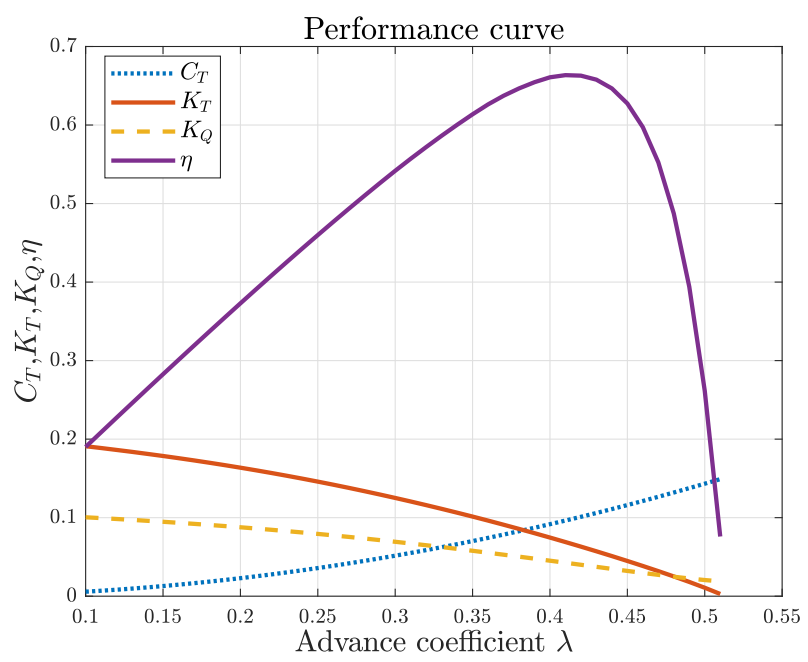

Figure 16: Steady-state performance curves as a function of advance coefficient $\lambda$ for the scaled cycloidal propeller used to simulate maneuvers of the cycloidal propeller-augmented UUV in the present work. The propeller phase $\gamma$ is $96^{\circ} . K_{T}, K_{Q}$ and $\eta$ are the thrust coefficient, torque coefficient and hydrodynamic efficiency respectively. $\quad C_{T}$ is the required thrust coefficient for motion in surge and is essentially the non-dimensionalized drag force. $C_{T}=K_{T}$ at $\lambda=0.38$ with $\eta \approx 0.65$. 


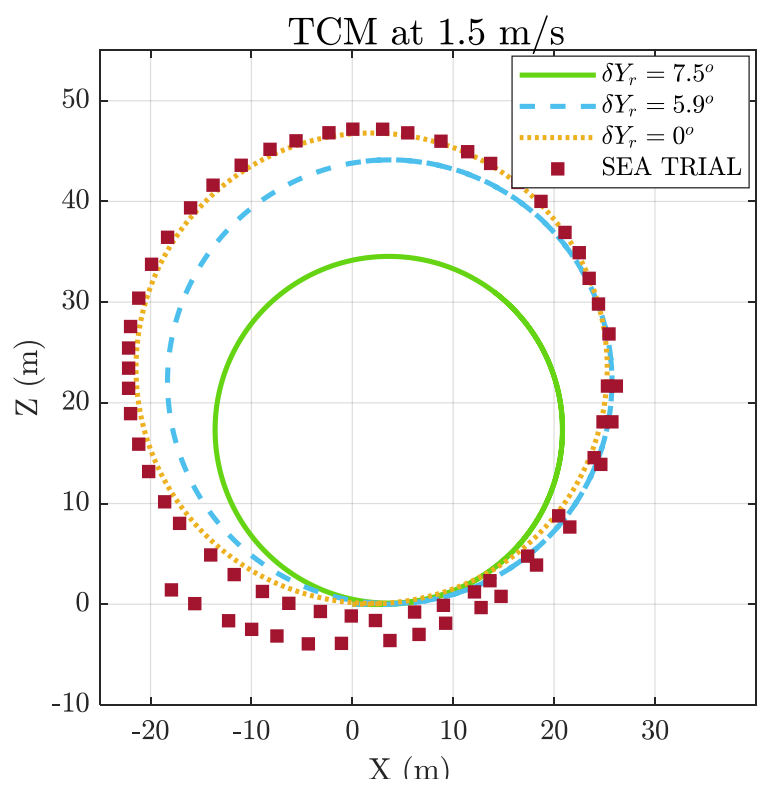

Figure 17: Simulated trajectories of a turning circle maneuver for the cycloidal propeller-augmented UUV in Figure 15, at a target speed of $1.5 \mathrm{~m} / \mathrm{s}$, in the port direction. Rudder deflection is given by $\delta \mathrm{Y}_{r} . \delta \mathrm{Y}_{r}=5.9^{\circ}$ and $\delta \mathrm{Y}_{r}=7.5^{\circ}$ are the two cases in which the cycloidal propellers only provide the thrust and the rudder provides the steering moments to execute the turn. $\delta \mathrm{Y}_{r}=0^{\circ}$ is the case in which the cycloidal propeller-augmented UUV describes a circle similar to that in the sea-trial in Figure 13. Here, the rudder is undeflected and the cycloidal propeller provides thrust and steering moments for the turning circle maneuver.

Figure 16 shows the steady-state characteristic curves for the scaled cycloidal propeller. We see that the cycloidal propeller has a maximum efficiency of $\sim 66 \%$ at an advance coefficient of $\lambda \sim 0.42$. To determine an efficient operating point, a required thrust coefficient $C_{T}$ is calculated as follows,

$$
C_{T}=\frac{0.25 \times \text { Drag }}{\frac{1}{2} \rho d b u^{2}}
$$

Drag is the Explorer AUV drag at a surge speed $V_{\infty} . \quad C_{T}$ is the non-dimensionalized drag which should be overcome by a single cycloidal propeller (hence the 0.25 multiplier). In Figure 16 we see that the $K_{T}$ (available non-dimensional thrust) and $C_{T}$ (required non-dimensional thrust) lines intersect at $\lambda=0.38$ and $\eta \sim 0.65$. This is the preferable design point of the scaled cycloidal propeller. Since the scaled cycloidal propeller is yet to be fully optimized, it has a lower hydrodynamic efficiency as compared to its commercial variants such as the Voith Schneider cycloidal propellers (hydrodynamic efficiency $\sim 73 \%$, Voith [2019]) found on full scale ships. Further consideration and optimization of parameters such as blade geometry and material, propeller size, number of blades, mission profile and propeller configuration, can improve the propeller performance, and mechanical reliability of the system. In the simulations to follow, the $\mathrm{X}, \mathrm{Y}$ and $\mathrm{Z}$ axes represent the surge, heave and sway degrees-of-freedom respectively.

\section{Turning circle maneuver at $1.5 \mathrm{~m} / \mathrm{s}$}

Table 6: Turning circle maneuver comparison at a target speed of $1.5 \mathrm{~m} / \mathrm{s}$ (Figure 17). Cycloidal propeller rotation speed $N$ and corresponding phase $\gamma$ required to execute the maneuvers are listed along with the steady-state turning radius $R_{T, s} . R_{T, s}$ in the sea-trial was $24.2 \mathrm{~m} . P$ lists the total power to be provided to the propellers.

\begin{tabular}{cccccc}
\hline Case & $\mathrm{CP}$ & $\begin{array}{c}\mathrm{N} \\
(\mathrm{RPM})\end{array}$ & $\begin{array}{c}\gamma \\
\left({ }^{\circ}\right)\end{array}$ & $\begin{array}{c}R_{T, s} \\
(\mathrm{~m})\end{array}$ & $\begin{array}{c}\mathrm{P} \\
(\mathrm{W})\end{array}$ \\
\hline$\delta \mathrm{Y}=7.5^{\circ}$ & $1-4$ & 196 & 97 & 17 & $4 \times 80$ \\
\hline$\delta \mathrm{Y}=5.9^{\circ}$ & $1-4$ & 196 & 97 & 22 & $4 \times 80$ \\
\hline$\delta \mathrm{Y}=0^{\circ}$ & 2,4 & 310 & 96 & 23 & $2 \times 535$ \\
& 1,3 & 65 & 295 & & $2 \times 36$ \\
\hline
\end{tabular}

The objective of this TCM comparison is to show that a cycloidal propeller-augmented UUV can execute turns similar to those of a screw propeller-control fin driven UUV, when solely propelled and maneuvered by cycloidal propellers, at a high UUV cruise speed.

Figure 17 compares the TCM trajectories of the screw propeller-control fin UUV from the sea-trial, to that of the cycloidal propeller-augmented UUV from the simulation. $\delta \mathrm{Y}_{r}=5.9^{\circ}$ and $\delta \mathrm{Y}_{r}=7.5^{\circ}$ are two cases in which the cycloidal propellers only provide the thrust necessary to maintain a steady surge speed of $1.5 \mathrm{~m} / \mathrm{s}$, while the rudder provides the steering to execute the turn. A larger fin deflection of $\delta \mathrm{Y}_{r}=7.5^{\circ}$ enables a sharper turn with a smaller turn radius. In the $\delta \mathrm{Y}_{r}=0^{\circ}$ case the cycloidal propeller-augmented UUV describes a circle similar to that in the sea-trial used for validating the six-DOF model. In this case, the cycloidal propeller provides thrust and steering moments for the TCM. As can be seen in Table 6, this comes at the cost of an appreciably higher power. The steering moments require high cycloidal propeller rotation speed, leading to a high power draw. For this test case, the cycloidal propeller rotation speed and phase were selected in a way that provided the same thrust and turning moment as in the screw propeller-control fin only case. Since the UUV must yaw toward the port side, rotation speed of 
the cycloidal propellers on the starboard side is larger than that on the other side in order to produce a thrust differential and turning moment.

\section{U-turn at $0.1 \mathrm{~m} / \mathrm{s}$}

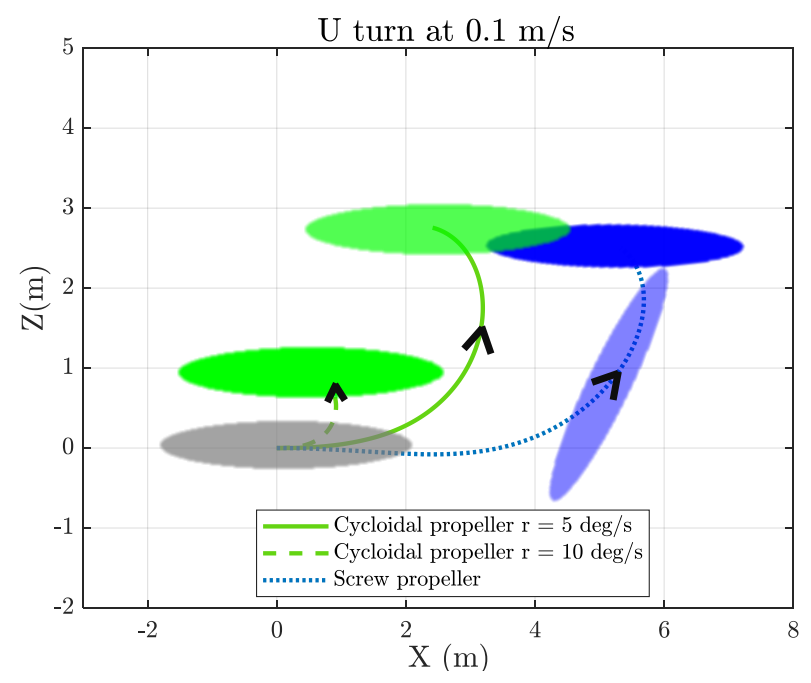

Figure 18: A comparison of a $0.1 \mathrm{~m} / \mathrm{s} 180^{\circ}$ turn, between a cycloidal propeller-augmented (in green) and screw propeller-control fin driven (in blue) UUV. In each case shown above, the UUV turns at high drift angles. This is shown only for the screw propeller case for the sake of clarity. The rudder angle $\delta \mathrm{Y}_{r}$ is $30^{\circ}$ and $0^{\circ}$ in the screw and cycloidal propeller cases respectively. The corresponding time taken to complete the maneuver is $95 \mathrm{~s}$ and $18 \mathrm{~s}$ (at a turn rate of $10 \mathrm{deg} / \mathrm{s}$ ) respectively. The gray ellipsoid represents a contour and the starting position for both UUVs. Since the rudder steering forces $\propto$ speed $^{2}$, at the low cruise speed of $0.1 \mathrm{~m} / \mathrm{s}$ the flow over the rudder is too slow and it sluggishly maneuvers the UUV. On the other hand, the cycloidal propellers rely on their rotation speed $\omega$ to generate flow over the blades. Hence, a sufficiently high rotation speed can enable a faster and more compact turn, even at a low cruise speed.

A U-turn at $0.1 \mathrm{~m} / \mathrm{s}$ is a low-speed maneuver a UUV may utilize when performing inspection operations in restricted waters or circling a point of interest for extended imaging and data collection. For this comparison, rudder deflection in the screw propeller-control fin driven UUV is set to $30^{\circ}$. In the cycloidal propeller-augmented UUV, two turning rates $r$ are considered, a higher yaw rate of 10 $\mathrm{deg} / \mathrm{sec}$ and a lower yaw rate of $5 \mathrm{deg} / \mathrm{sec}$. The rudders are undeflected.

Figure 18 compares the low speed $180^{\circ}$ turn trajectories for three cases. The rudder angle $\delta \mathrm{Y}_{r}$ is $30^{\circ}$ and $0^{\circ}$ in the screw and cycloidal propeller cases respectively. The gray ellipsoid represents a contour and the starting point for both UUVs. The blue ellipsoid is the screw propeller-control fin UUV while the green ellipsoid is the cycloidal propeller-augmented UUV. We see that with a screw propeller, the UUV executes a slower turn that occupies a much larger area as compared to when maneuvered using cycloidal propellers. As can be seen in Table 8, its response time of 95 seconds $(r=1$ $\mathrm{deg} / \mathrm{s})$ is a lot more than the 18 seconds $(\mathrm{r}=10 \mathrm{deg} / \mathrm{s})$ with cycloidal propellers. This is because at the low UUV cruise speed of $0.1 \mathrm{~m} / \mathrm{s}$, the fin steering forces $(\propto$ speed $^{2}$ ) are too low to allow swift response, despite a rudder deflection as large as $30^{\circ}$. On the other hand, cycloidal propeller maneuvering forces being much less dependent on UUV speed, continue to provide effective steering moments to the UUV, leading to a quicker and more compact response, an attribute desirable in restricted waters, swarming and teaming. Quite naturally, higher turning rates will require higher propeller rotation speeds and power draw.

Table 7: $180^{\circ}$ turn comparison for a target speed of 0.1 $\mathrm{m} / \mathrm{s}$ (Figure 18). $\quad N$ is the cycloidal propeller rotation speed and $\gamma$ is the corresponding cycloidal propeller blade phase. $t_{U}$ is the time taken to execute the maneuver. $P$ is the total power to be provided to the propellers while $E$ is the estimated energy consumed by the cycloidal propellers while the maneuver is executed.

\begin{tabular}{ccccccc}
\hline $\mathrm{r}$ & $\mathrm{CP}$ & $\mathrm{N}$ & $\gamma$ & $t_{U}$ & $\mathrm{P}$ & $\mathrm{E}$ \\
$(\% / s)$ & & $(\mathrm{RPM})$ & $\begin{array}{c}\left.{ }^{\circ}\right) \\
(\mathrm{s})\end{array}$ & $(\mathrm{W})$ & $(\mathrm{W}-\mathrm{h})$ \\
\hline \multirow{2}{*}{10} & 2,4 & 165 & 97 & 18 & $2 \times 110$ & $2 \times 0.55$ \\
& 1,3 & 150 & 278 & & $2 \times 87$ & $2 \times 0.44$ \\
\hline \multirow{2}{*}{5} & 2,4 & 87 & 97 & 47 & $2 \times 10.53$ & $2 \times 0.06$ \\
& 1,3 & 25 & 250 & & $2 \times 2$ & $2 \times 0.01$ \\
\hline
\end{tabular}

\section{Heave at $0.5 \mathrm{~m} / \mathrm{s}$}

In UUVs, a controlled heave motion is necessary to avoid collisions when operating near the sea bed. UUVs without tunnel/azimuthal thrusters, like the Explorer AUV, utilize control planes (horizontal stern planes and/or dive planes) to alter depth. They do so by pitching up or down and moving along a curved trajectory (Issac [2011]). Being maneuvered by control planes, the UUV must have a finite surge velocity for the control planes to be effective. As a result, while heaving, the UUV will also advance in surge. Such a luxury of space may not always be available, especially when descending narrow passages during ocean surveys. 


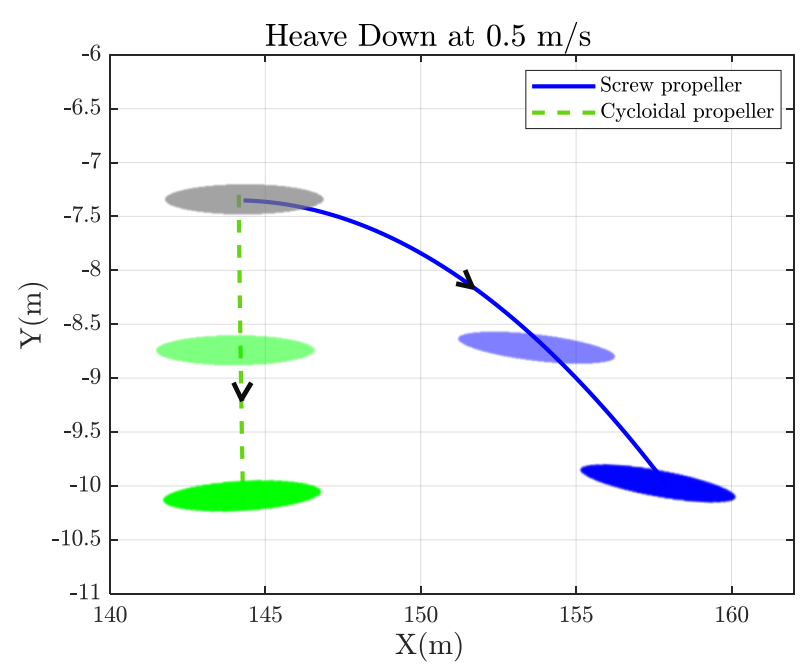

Figure 19: A $0.5 \mathrm{~m} / \mathrm{s}$, three meter heave down (Y-axis) comparison between cycloidal and screw propeller driven underwater vehicles (UVs). Both UVs start at the same point (dotted ellipse). The cycloidal propeller driven UV (dashed trajectory) heaves straight down by vectoring its thrust downwards. The screw propeller and control fin only driven UV (solid trajectory) pitches down and moves with a finite surge speed $(0.5 \mathrm{~m} / \mathrm{s})$ for the fins to be effective in heaving the UV, causing the UV to significantly advance in surge $(\Delta X \sim 20 \mathrm{~m})$. Such a luxury of space may not always be available when operating in restricted spaces (for eg. near underwater infrastructure).

Table 8: Heave comparison at $0.5 \mathrm{~m} / \mathrm{s}$ (Figure 19). $N$ is the cycloidal propeller rotation speed and $\gamma$ is the corresponding cycloidal propeller blade phase. $P$ is the total power to be provided to the propellers while $E$ is the estimated energy consumed by the cycloidal propellers while the maneuver is executed.

\begin{tabular}{cccccc}
\hline $\mathrm{CP}$ & $\mathrm{N}$ & $\gamma$ & $t_{H}$ & $\mathrm{P}$ & $\mathrm{E}$ \\
& $(\mathrm{RPM})$ & $(\mathrm{deg})$ & $(\mathrm{s})$ & $(\mathrm{W})$ & $(\mathrm{W}-\mathrm{h})$ \\
\hline 1,2 & 243 & 96 & 6 & $2 \times 329$ & $2 \times 0.55$ \\
3,4 & 183 & 96 & & $2 \times 134$ & $2 \times 0.22$ \\
\hline
\end{tabular}

Figure 19 shows a comparison between heave trajectories for a screw propeller-control fin steered UUV and a cycloidal propeller-augmented UUV. The gray ellipsoid represents a contour and the starting point for the UUV in both cases. The motion between depths of seven meters and 10 meters is analyzed. The screw propeller driven UUV (in blue) moves at $0.5 \mathrm{~m} / \mathrm{s}$ and pitches down by $\sim 6^{\circ}$ to gain depth. Time-logged positions and pitch angles recorded in Issac [2011] are used to recreate the
UUV motion. The UUV took 30 seconds to descend the $3 \mathrm{~m}$ depth and advanced close to $15 \mathrm{~m}$ in surge in the process. This is not desirable in restricted waters. On the other hand, the cycloidal propeller-augmented UUV (in green) is capable of descending straight down, simply by vectoring thrust in the downward direction. Moving at $0.5 \mathrm{~m} / \mathrm{s}$, it takes 6 seconds to move to a depth of 10 meters. More importantly, it does not advance in surge. It is also seen in the figure that perfect trim is not attained with the cycloidal propellers and the UUV pitches up to $5^{\circ}$, pointing towards the need for a control system that can vector cycloidal propeller thrust to execute such a maneuver.

\section{CONCLUSION AND FUTURE WORK}

The present work investigated a novel concept of using cycloidal propellers to augment the maneuvering of an unmanned underwater vehicle (UUV). Cycloidal propellers are cross-flow propellers that utilize $360^{\circ}$ thrust vectoring to provide agile and compact maneuvering to surface vessels like tug boats and double ended ferries, but have yet to be utilized on present-day unmanned and autonomous marine vehicles. Through simulations, this paper analyzed and compared the maneuvering characteristics of an unmanned underwater vehicle (UUV) driven by a screw propeller and control fins only, to that of one augmented with cycloidal propellers. In the cycloidal propeller-augmented UUV, four cycloidal propellers (two each on port and starboard sides) were added to an existing screw propeller-control fin setup. These cycloidal propellers are envisioned to be retractable. At a low cruise speed, only the cycloidal propellers provided the thrust for propulsion and maneuvering. At higher cruising speeds, only the screw propeller provided the thrust for propulsion while the control planes maneuvered the UUV. Such a UUV configuration can be of use in a UUV which will spend a larger portion of its mission duration in getting to the point of interest. During this time, the cycloidal propellers can be retracted into the UUV hull and the screw propellers and control fins can be used to ensure fast and efficient travel. A relatively shorter duration may be spent while collecting data at a low cruise speed, during which time the cycloidal propellers can be extended out of the UUV hull and used for accurate positioning and swift maneuvering of the UUV.

The maneuvers simulated were a $1.5 \mathrm{~m} / \mathrm{s}$ turning circle maneuver, a $0.1 \mathrm{~m} / \mathrm{s} 180^{\circ}$ turn and a $0.5 \mathrm{~m} / \mathrm{s}$ heave maneuver. A previously validated cycloidal propeller hydrodynamic model was used to simulate the cycloidal propeller forces. A six-DOF reduced order model was developed to predict the trajectory of the underwater vehicle. This reduced order motion solver necessarily 
accounted for the non-linear and coupled effects of the hydrodynamic, hydrostatic and Coriolis-Centripetal loads that an underwater vehicle experiences during service. This is important given the higher density of water and the bulky make of an underwater vehicle which can result in large, coupled and destabilizing motions that severely affect vehicle maneuvering and control. In addition to being computationally cheaper and faster than traditional CFD methods used to predict underwater vehicle trajectories, the reduced order model is also easy to integrate with controller design software.

The $0.1 \mathrm{~m} / \mathrm{s} 180^{\circ}$ turn showed that while control fins are less effective in steering a UUV cruising at a low speed (steering force $\propto$ speed $^{2}$ ), cycloidal propellers can continue to provide compact and high steering rates by rotating at sufficiently high speeds and producing a differential thrust and moment on the port and starboard sides of the UUV. In the $0.5 \mathrm{~m} / \mathrm{s}$ heave down maneuver simulated, the potential for cycloidal propellers to provide decoupled motion through thrust vectoring was highlighted. Unlike with a screw propeller-control fin driven UUV, the cycloidal propeller-augmented UUV did not descend by pitching down and advancing in surge (i.e. coupling heave with surge). Instead, the cycloidal propellers vectored thrust downwards to make the UUV gain depth. Therefore, thrust vectoring of cycloidal propellers in unmanned and autonomous marine vehicles can enable controlled, decoupled, swift, and compact maneuvering in all six-DOF, while potentially overcoming disturbances like waves and currents, and extreme flow conditions like cavitation and ventilation. This is critical in operations conducted at low cruise speed and in restricted waters, such as underwater infrastructure inspection, minesweeping and sea-keeping.

It should be kept in mind that cycloidal propeller performance and power consumption in a low speed maneuver is affected by the degree-of-freedom and aggressivity of the maneuver, and the required propeller speed. A swift $180^{\circ}$ turn of a small radius will have a high power draw to overcome the large hydrodynamic resistance due to a high turn rate, large side-slip angle and high propeller speed. Similarly, a decoupled heave maneuver will require a high power draw since the UUV will usually present a larger profile area in this degree-of-freedom. However, if the cycloidal propeller enabled maneuvers are made less aggressive and as slow as those enabled by the screw propeller-control plane combination, the rotation speed and power draw required by the cycloidal propeller would drop significantly (power $\propto$ speed $^{3}$ ), without coupling and affecting the compactness of the maneuvers.

The next step in the present work is to design a robust control system to track and vector thrust of cycloidal propellers, and obtain the required maneuvering in a marine vehicle. The control system must consider the hydrodynamic coupling between the propeller speed, phase and eccentricity, and select an efficient operating point for the cycloidal propeller. The control system must also estimate the flow conditions over the propeller in order to reject measurement noise, attenuate exogenous disturbances like waves and currents and compensate for extreme flow conditions like cavitation and ventilation (Desai et al.). To aid development of such a control system, it is also planned to add the physics associated with cavitation and ventilation into the reduced-order hydrodynamic model developed by Halder et al. [2017] for cycloidal propellers. On the maneuvering side, waves and currents will be modeled into the six-DOF reduced order motion solver. Cavitation and ventilation can occur on fast-moving cycloidal propeller blades operating close to the surface, leading to vibration and noise, and a sudden loss of propeller force and vehicle control. Waves will add wave drag, and frequency dependence in fluid-added mass and damping of the marine vehicle. Coupling the above control system with the six-DOF motion solver can accelerate exploration of cycloidal propellers for the preliminary design of advanced autonomous marine vehicles and smart platforms for increasingly important applications like payload delivery, equipment deployment, ocean surveying, station keeping, sea-keeping, swarming and teaming.

Real-world implementation of cycloidal propellers for unmanned marine vehicles and smart platforms is not guaranteed, and requires examination of decision parameters such as the vehicle size, propeller geometry and power requirements, battery technology, blade design, payload, mission requirements, and environmental conditions. Detailed CFD analysis for cycloidal propeller performance, and estimation of propeller-propeller and propeller-hull interaction effects, should be carried out. If found feasible for the technology of tomorrow, cycloidal propellers could usher in a new era for unmanned marine vehicles.

\section{ACKNOWLEDGEMENTS}

The authors are grateful for the funding provided for this study by the Office of Naval Research (ONR) grant no. N00014-18-S-B001 managed by Troy Hendricks.

\section{REFERENCES}

Moble Benedict, Manikandan Ramasamy, and Inderjit Chopra. Improving the aerodynamic performance of micro-air-vehicle-scale cycloidal rotor: an experimental approach. Journal of Aircraft, 47 (4):1117-1125, 2010. 
Moble Benedict, Raghav Gupta, and Inderjit Chopra. Design, development, and open-loop flight-testing of a twin-rotor cyclocopter micro air vehicle. Journal of the American Helicopter Society, 58(4):1-10, 2013a.

Moble Benedict, Tejaswi Jarugumilli, and Inderjit Chopra. Effect of rotor geometry and blade kinematics on cycloidal rotor hover performance. Journal of Aircraft, 50(5):1340-1352, $2013 \mathrm{~b}$.

Moble Benedict, Tejaswi Jarugumilli, Vinod Lakshminarayan, and Inderjit Chopra. Effect of flow curvature on forward flight performance of a micro-air-vehicle-scale cycloidal-rotor. AIAA Journal, 52(6):1159-1169, 2014.

M. Desai, A. Halder, M. Benedict, and Y.L. Young. A control scheme for $360^{\circ}$ thrust vectoring of cycloidal propellers. Under review.

Dietmer Deter. Principle aspects of thruster selection. In Dynamic Positioning Conference, Oct. 21-22, Houston, USA. Marine Technology Society, 1997.

Jason Evans and Meyer Nahon. Dynamics modeling and performance evaluation of an autonomous underwater vehicle. Ocean Engineering, 31(14-15):1835-1858, 2004.

Odd M Faltinsen. Hydrodynamics of high-speed marine vehicles. Cambridge University press, 2005.

Fleetmanager. Fleetmanager, Advanced Concept and System Architecture, France, 2020. www . underwater-gps.com, Last accessed on 2019-08-28.

Thor I Fossen. Handbook of marine craft hydrodynamics and motion control. John Wiley \& Sons, 2011.

Gwyn Griffiths. Technology and applications of autonomous underwater vehicles, volume 2 . CRC Press, 2002.

Atanu Halder and Moble Benedict. Nonlinear aeroelastic modeling of cycloidal rotor in forward flight. AHS Technical Meeting on Aeromechanics Design for Transformative Vertical Lift, San Francisco, California, January 20-22, 2018, 2018.

Atanu Halder, Carolyn Walther, and Moble Benedict. Unsteady hydrodynamic modeling of a cycloidal propeller. In Fifth International Symposium on Marine Propulsion Smp, volume 17, 2017.

Atanu Halder, Carolyn Walther, and Moble Benedict. Hydrodynamic modeling and experimental validation of a cycloidal propeller. Ocean Engineering, 154: 94-105, 2018.
RC Hibbeler and Kai Beng Yap. Mechanics for Engineers: Dynamics, volume 38. Pearson Education South Asia Pte Ltd, 2012.

ISE. International Submarine Engineering Ltd. https: //ise.bc.ca/wp-content/uploads/2017/ 12 /ExplorerSpecSheet.pdf, Last accessed on 2019-10-30.

Manoj Thomas Issac. Analysis of full-scale sea-trials manoeuvring data and development and validation of a motion-simulation model for the AUV MUN Explorer. $\mathrm{PhD}$ thesis, Memorial University of Newfoundland, 2011.

D Jürgens, $\mathrm{M}$ Palm, and A Brandner. Comparative investigation on influence of the positioning time of azimuth thrusters on the accuracy of dp. In Dynamic Positioning Conference, Houston, 2012.

D Jürgens, M Palm, and Voith Turbo. Influence of thruster response time on dp capability by time-domain simulations. In Marine Technology Society-DP Conference October, 2017.

Dirk Jürgens, Torsten Moltrecht, and Marcel Flipse. Cycloidal propulsion-the quiet maneuvering propulsion for large motor yachts. 2004.

D Jurgins and H Heinke. Voith schneider propeller (vsp)-investigations of the cavitation behavior. In First International Symposium on Marine Propulsors (SMP'09), June, 2009.

P. Koschorrek, C. Siebert, L. Theilen, O. Detlefsen, M. Palm, K. Mach, M. Steinwand, T. Jeinsch, and M. Abdel-Maksoud. Dynamic positioning with voith schneider propeller: Experimental system validation with a model-scale offshore supply vessel. In $\underline{2017}$ 22nd International Conference on Methods and Models in Automation and Robotics (MMAR), pages 7-12, 2017.

John Nicholas Newman. Marine hydrodynamics. MIT press, 2018.

R. O'Rourke. Navy Large Unmanned Surface and Undersea Vehicles: Background and Issues for Congress. Amazon Digital Services LLC - Kdp Print Us, 2019. ISBN 9781075833274. URL https://books.google.com/books?id= SF $3 \mathrm{HxgEACAAJ}$.

Ronald O'Rourke. Unmanned vehicles for US Naval forces: Background and issues for congress. 2006.

Ronald O'Rourke. Navy Littoral Combat Ship/frigate (LCS/FFGX) Program: Background and Issues for Congress. Congressional Research Service, 2017. 
Michael Palm, Dirk Jürgens, and David Bendl. Numerical and experimental study on ventilation for azimuth thrusters and cycloidal propellers. In Proc. 2nd Int. Symp. Marine Propulsors smp, volume 11, 2011.

Bernard T Roesler, Manaure Francsiquez, and Brenden P Epps. Design and analysis of trochoidal propulsors using nonlinear programming optimization techniques. In ASME 2014 33rd International Conference on Ocean, Offshore and Arctic Engineering, pages V08BT06A032-V08BT06A032. Citeseer, 2014.

Bernard T Roesler, Malia L Kawamura, Eric Miller, Matthew Wilson, Jonathon Brink-Roby, Eric Clemmenson, Matthew Keller, and Brenden $\mathrm{P}$ Epps. Experimental performance of a novel trochoidal propeller. Journal of Ship Research, 60(1):48-60, 2016.

Voith. Voith Schneider Propeller VSP, 2019. WwW . voith. com, Last accessed on 2019-09-19.

Commons Wikimedia. File:vspreal.jpg — wikimedia commons, the free media repository, 2014. URL https://commons.wikimedia.org/ w/index.php?title=File:VSPreal.

jpg\&oldid=119474711. [Online; accessed 21-July-2020].

Commons Wikimedia. File:brosen propelersterntychy.jpg - wikimedia commons, the free media repository, 2019. URL https://commons.wikimedia. org/w/index.php?title=File:Brosen_ propelersterntychy · jpg\&oldid=

351643558 . [Online; accessed 21-July-2020].

Yin Lu Young. Hydroelastic response of lifting bodies in separated flows. NATO-AVT-307: Symposium on Separated Flow: Prediction, Measurement and Assessment for Air and Sea Vehicles, Oct. 7-9, Trondheim, Norway, 2019.

Yin Lu Young, Casey M Harwood, F Miguel Montero, Jacob C Ward, and Steven L Ceccio. Ventilation of lifting bodies: Review of the physics and discussion of scaling effects. Applied Mechanics Reviews, 69(1), 2017.

\section{APPENDIX}

\section{Rotation matrices}

$\psi, \phi$ and $\theta_{v}$ are the Euler angles of orientation of 1'2'3' in the inertial coordinate system 123. Eq. (18) and (19) show the effective rotation matrix $R$ Fossen [2011] that orients $\vec{x}$ - a vector in the vehicle frame 1 '2'3' to $\vec{X}$ - a vector in the inertial frame 123 .

$$
R=\left[\begin{array}{ccc}
c \psi c \theta_{v} & -s \psi c \phi+c \psi s \theta_{v} s \phi & s \psi s \phi+c \psi c \phi s \theta_{v} \\
s \psi c \theta_{v} & c \psi c \phi+s \phi s \theta_{v} s \psi & -c \psi s \theta_{v}+s \psi s \theta_{v} c \phi \\
-s \theta_{v} & c \theta_{v} s \phi & c \theta_{v} c \phi
\end{array}\right]
$$

where c. $=\cos ($.$) and \mathrm{s} .=\sin ($.$) .$

$$
X_{i}=R_{i j} x_{j} ; i, j=1,2,3
$$

\section{Equation of motion matrices}

Eq. (20) - (30) list the expansions for the matrices used in the six-DOF equations of motion.

\section{Structural mass matrix $M_{S}$}

$$
\boldsymbol{M}_{\boldsymbol{S}}=\left[\begin{array}{cc}
m I_{3 \times 3} & -m S\left(r_{g}^{b}\right) \\
m S\left(r_{g}^{b}\right) & m I_{b}
\end{array}\right]
$$

$\mathrm{m}$ is the wet mass of the vehicle. $I_{3 \times 3}$ is the identity matrix while $I_{b}$ is the vehicle rotational inertia matrix in the body-fixed frame 1'2'3 defined as,

$$
I_{b}=\left[\begin{array}{ccc}
I_{1^{\prime} 1^{\prime}} & -I_{1^{\prime} 2^{\prime}} & -I_{1^{\prime} 3^{\prime}} \\
-I_{2^{\prime} 1^{\prime}} & I_{2^{\prime} 2^{\prime}} & -I_{2^{\prime} 3^{\prime}} \\
-I_{3^{\prime} 1^{\prime}} & -I_{3^{\prime} 2^{\prime}} & I_{3^{\prime} 3^{\prime}}
\end{array}\right]
$$

$r_{g}^{b}$ is the position vector, in the body-fixed frame $1^{\prime} 2^{\prime} 3^{\prime}$, of the point about which moments are evaluated. This is usually the center of gravity of the vehicle. $S$ is defined as follows for $\boldsymbol{k}=\left[k_{1}, k_{2}, k_{3}\right]$,

$$
S(\boldsymbol{k})=\left[\begin{array}{ccc}
0 & -k_{3} & k_{2} \\
k_{3} & 0 & -k_{1} \\
-k_{2} & k_{1} & 0
\end{array}\right]
$$

$\underline{\text { Fluid added mass matrix } M_{A}}$

$$
M_{A_{i j}}=\left(X_{i}\right)_{\dot{v}_{j}}
$$

$\left(X_{i}\right)_{\dot{v}_{j}}=\frac{\partial X_{i}}{\partial \dot{v}_{j}}$ is the hydrodynamic derivative for the fluid added mass as per SNAME conventions. $\dot{v}$ is the acceleration vector of the vehicle in the body-fixed frame $1^{\prime} 2^{\prime} 3^{\prime}$. $i, j=1,2,3$ for translational and $i, j=4,5,6$ for rotation along axes $1^{\prime}, 2^{\prime}$ and $3^{\prime}$.

$\underline{\text { Structural Coriolis-centripetal matrix } C_{S}}$

$$
\boldsymbol{C}_{\boldsymbol{S}}=\left[\begin{array}{cc}
0_{3 \times 3} & -S\left(M_{11} v_{1}+M_{12} v_{2}\right) \\
-S\left(M_{11} v_{1}+M_{12} v_{2}\right) & -S\left(M_{21} v_{1}+M_{22} v_{2}\right)
\end{array}\right]
$$


$v_{1}=[u, v, w]^{T}$ and $v_{2}=[p, r, q]^{T}$. Here, $M_{S}$ has been split into four $3 \times 3$ sub-matrices,

$$
M_{S}=\left[\begin{array}{ll}
M_{11} & M_{12} \\
M_{21} & M_{22}
\end{array}\right]
$$

Fluid Coriolis-centripetal matrix $C_{A}$

$$
\boldsymbol{C}_{\boldsymbol{A}}=\left[\begin{array}{cc}
0_{3 \times 3} & -S\left(A_{11} v_{1}+A_{12} v_{2}\right) \\
-S\left(A_{11} v_{1}+A_{12} v_{2}\right) & -S\left(A_{21} v_{1}+A_{22} v_{2}\right)
\end{array}\right]
$$

Here, $M_{A}$ has been split into four $3 \times 3$ sub-matrices,

$$
\boldsymbol{M}_{\boldsymbol{A}}=\left[\begin{array}{ll}
A_{11} & A_{12} \\
A_{21} & A_{22}
\end{array}\right]
$$

$\underline{\text { Non-linear damping matrix } D_{n l}}$

$$
\begin{gathered}
\boldsymbol{D}_{n l_{i j}}=\left(X_{i}\right)_{v_{j}\left|v_{j}\right|} \\
\left(X_{i}\right)_{v_{j}\left|v_{j}\right|}=\frac{\partial X_{i}}{\partial\left(v_{j}\left|v_{j}\right|\right)} \text { is the hydrodynamic }
\end{gathered}
$$
derivative for the non-linear damping forces and moments as per SNAME conventions. $i, j=1,2,3$ for translational and $i, j=4,5,6$ for rotation along axes $1^{\prime}, 2^{\prime}$ and $3^{\prime}$.

$\underline{\text { Linear damping matrix } D_{l}}$

$$
\boldsymbol{D}_{l_{i j}}=\left(X_{i}\right)_{v_{j}}
$$

$\left(X_{i}\right)_{v_{j}}=\frac{\partial X_{i}}{\partial v_{j}}$ is the hydrodynamic derivative for the linear damping forces and moments as per SNAME conventions. $i, j=1,2,3$ for translational and $i, j=4,5,6$ for rotation along axes $1^{\prime}, 2^{\prime}$ and $3^{\prime}$.

$\underline{\text { Hydrostatic forces and moments } g_{i}}$

$$
\boldsymbol{g}_{i}=\left[\begin{array}{c}
(W-B) \sin \theta_{v} \\
-(W-B) \sin \phi \cos \theta_{v} \\
-(W-B) \cos \phi \cos \theta_{v} \\
-\left(W x_{3}^{G}-B x_{3}^{B}\right) \cos \phi \cos \theta_{v}-\left(W x_{2}^{G}-B x_{2}^{B}\right) \sin \phi \cos \theta_{v} \\
-\left(W x_{2}^{G}-B x_{2}^{B}\right) \sin \theta_{v}+\left(W x_{1}^{G}-B x_{1}^{B}\right) \cos \phi \cos \theta_{v} \\
-\left(W x_{1}^{G}-B x_{1}^{B}\right) \sin \phi \cos \theta_{v}-\left(W x_{3}^{G}-B x_{3}^{B}\right) \sin \theta_{v}
\end{array}\right]
$$

$W$ is the self weight of the vehicle while $B$ is the buoyancy force acting on the vehicle. $x^{B}$ and $x^{G}$ pertains to the center of buoyancy and center of gravity coordinates respectively, while $\theta_{v}$ and $\phi$ are the pitch and roll angles respectively.

\section{DISCUSSION AND AUTHORS' REPLY}

We would like to first thank all the reviewers for their time and feedback. The authors' reply are given in italic font after comments presented by each reviewer. The contents of this paper have been revised based on the reviews received.

\section{Dr. John Dzielski}

Stevens Institute of Technology, Davidson Laboratory New Jersey, USA

Thank you for a very interesting paper describing a novel application of CP technology to a UUV. I like to read papers that include well organized descriptions of the physics associated with complicated hydrodynamic mechanisms. It seems that the technology that would implement a comparable functionality to the one described in the paper would be a tunnel thruster. Several UUVs (e.g. NPS Phoenix) have used tunnel thrusters in the same configuration as described in the paper. Would you discuss why this technology was not considered for comparison?

In addition to tunnel thrusters, one could achieve a similar effect with a bow-mounted vertical rudder or even a propeller with a helicopter-like main propeller swashplate (e.g. NAVOCEANO Lazarus). Would you comment on any advantage or disadvantages of these approaches relative to the $\mathrm{CP}$ concept under different scenarios. Please consider the tunnel thruster in the discussion if you haven't already addressed this.

Would it be possible to implement something like a collective on a helicopter to produce thrust along the axis of rotation? I'm thinking of a non-zero theta, independent of beta. What would it take? You may also explain why this is a stupid question.

Authors' response - Thank you for the positive comments. Indeed, tunnel thrusters can be used to augment maneuvering capabilities of a UUV driven by screw propeller(s) and control fins. However, while each cycloidal propeller can enable $360^{\circ}$ thrust vectoring, each tunnel thruster can only generate thrust in the direction of flow. Hence, tunnel thrusters are typically used to generate differential side force to facilitate yawing motion only for a UUV, while cycloidal propellers can be used to generate differential force to facilitate all six degrees of freedom (six-DOF: surge, heave, sway, yaw, roll, and pitch) for a UUV. However, cycloidal propellers are not efficient at high forward speeds, and hence this paper focuses on the efficacy of using cycloidal propellers to augment the thrust and maneuvering capability of a UUV 
driven by a propeller and control fins. We have modified the paper to include a brief review and comparison of different propulsion options.

Similar to the response to the comments about tunnel thrusters, the main attraction for the cycloidal propeller is its $360^{\circ}$ thrust vectoring ability. Bow-mounted vertical rudder is typically used to assist yawing motion only and does not facilitate six-DOF maneuvering. The cyclic and collective pitching of the blades on a helicopter-like main swashplate can deliver axial and transverse force to augment the motion of a baseline UUV (UUV with screw propeller and control fins only) in multiple DOF. However, using a single cyclic and collective pitch propeller (CCPP) may not provide decoupled attitude control in a UUV cruising at a low speed. This is because sufficiently large counter-moments may not be generated by the control fins (steering force $\propto$ speed $^{2}$ ) to eliminate the moment in roll, and control moments in other DOF, induced by the transverse thrust component of the CCPP. This is not the case with the cycloidal propeller-augmented UUV simulated in this paper. The four (retractable) cycloidal propellers, each with independent $360^{\circ}$ thrust vectoring capability, can control vehicle attitude while precisely maneuvering the UUV during a low speed operation in restricted waters.

Having two CCPPs, one each at the bow and stern, can reduce the coupled motion described above and enhance control in all six-DOF of the UUV. Alternatively, two large hub-to-diameter ratio CCPPs could wrap around the UUV hull (for eg. the US Navy's Tandem Propeller System) and provide six-DOF maneuvering. Two CCPPs may also eliminate the need for control fins, while augmenting the thrust provided by the screw propeller. In a UUV cruising at a high speed, a non-rotating CCPP could be used to effectively maneuver the UUV by simply changing the cyclic pitch of the blades. On the other hand, at a high cruise speed, cycloidal propellers are less efficient at producing thrust and are not the preferred option to maneuver the UUV. However, a tandem CCPP will wrap around the UUV hull and restrict the hull geometry to a circular cross-section. Additionally, two CСРP may occupy a large internal volume due to a (possibly) bulky machinery. Weight, mechanical reliability and system efficiency are other parameters that must also be analyzed. Another specific benefit of the amphibious vehicle design with four CPS shown in Fig. 1 is that they can be retracted to be covered within the wheels, to minimize drag when the CPs are not in use, and the wheels use the same drive train for land-based operation. Helicopter-like propellers cannot provide such capability. Comparison of the UUV with screw propeller and control fins was conducted for code validation purposes, and to demonstrate the enhanced maneuverability and agility with the CPs compared to conventional screw propeller and control fins. A brief comparison of various propulsion options has been added to the revised introduction.

Theta is the pitch angle of the propeller blade; it is the angle that the blade chord makes with the tangent to the blade orbit of rotation. Beta is the azimuthal angle along the circumference of this orbit of rotation. For the cycloidal propeller simulated in this paper, the blade span and the blade pitch axis always remain parallel to the propeller axis of rotation. Therefore, simply a non-zero theta, independent of beta, will not produce thrust along the propeller axis of rotation. The cycloidal propeller thrust will remain within the plain of the propeller disk. The propeller blade span should be made non-parallel to the propeller axis rotation in order to have a component of blade force, and hence propeller thrust, along the propeller axis of rotation. However, such an arrangement may require the blade to rotate outside the plane of the propeller disk, or 'flap', while pitching (cycloidally) at the same time. This will increase the mechanical complexity of the system.

A simpler alternative may be to mount the cycloidal propellers at an angle not orthogonal to the body axis. For example, the cycloidal propellers could be mounted at a $45^{\circ}$ angle to the heave axis. The propeller speed and phase can be adjusted in a manner so as to obtain a net force in the direction of the propeller axis, or any direction for that matter.

Prof. Dr.-Ing. Moustafa Abdel-Maksoud

Hamburg University of Technology (TUHH), Institute for Fluid Dynamics and Ship Theory (M8) - Hamburg, Germany

The paper addresses a very interesting and innovative application regarding the integration of cycloidal propeller in the design concept of UUV. The procedure for evaluating the hydrodynamic performance of cycloidal propellers under consideration of different operating conditions is well described. Although the integration of the four cycloidal propellers increases the functionality of UUV in regions with limited working space, the complexity of the vehicle increases considerably.

The consideration of the following aspects in the paper may help the reader to gain a better understanding of the paper:

- The main challenge in the development and implementation of the control system for the cycloidal propellers is the accurate estimation of the interaction parameters between the individual 
propulsion units and between the propulsion units and the vehicle hull. The interaction parameters vary with the UUV's speed and motion direction as well as with the environmental parameters such as local current characteristics und site topography. This aspect was hardly considered in the paper.

- The combination of one main propeller and four cycloidal propellers has many disadvantages. When the cycloidal propellers are not in operation, they will increase the total drag of the vehicle considerably, which will have a negative effect on the duration of the UUV's mission.

- The influence of number of blades of the cycloidal propellers is not investigated in the paper. Reducing the number of blades to two could reduce the resistance of the cycloidal propellers during shutdown time, since the blade profiles can be aligned parallel to the direction of flow, when the UUV travels long distances straight ahead. A reduction of the number of blades also has a positive effect on the efficiency of the cycloidal propellers.

I would like to congratulate the authors for their innovative work and their successful implementation and wish them every success in their future work.

Authors' response - Thank you for the positive comments. In this paper, the propeller-propeller and propeller-hull interactions have not been modeled and are neglected in the simulation study of the UUVs. Indeed, such interactions are important in the development and implementation of a robust control system for the $360^{\circ}$ thrust vectoring of each cycloidal propeller, and the agile maneuvering and control of the UUV. The above interactions will not only affect the hydrodynamic performance of the cycloidal propeller, but also the unsteady hydrodynamic forces on the UUV. In future, the parameters and hydrodynamic coefficients necessary to account for such effects can be found using CFD studies of the UUV and/or model scale experiments of the UUV in a towing tank. However, these coefficients and empirical corrections can change depending on the operating environment of the UUV. A stable and optimal observer, that accounts for modeling errors and uncertainties in these coefficients, sensor noise and disturbances such as water currents and waves, will be a necessary and an integral part of the cycloidal propeller control system. Hence, we have focused on the development of a $360^{\circ}$ thrust vectoring methodology for the cycloidal propeller subject to varying inflow speed and noise. A brief summary of the control method will be presented at the conference, while the details are presented in a paper that was recently submitted to IEEE (Desai et al.).

The cycloidal propellers are envisioned to be retractable. This has been clarified in the revised Cycloidal Propeller vs. Screw Propeller section of the paper. Hence, when not in operation, the cycloidal propellers are to be retracted and stowed inside wheel covers, as shown in Fig 3, so that they do not increase the total drag of the vehicle. In addition, the wheel covers function as wheels for land-based operation of the amphibious vehicle shown in Figure 3, and the wheels and cycloidal propeller are driven by the same drive train to minimize size and weight.

Indeed, reducing the number of blades from four to two will have a positive effect on the hydrodynamic efficiency of the cycloidal propeller. A two-bladed and horizontally oriented cycloidal propeller may in fact be used as a horizontal control plane to pitch and roll the vehicle by either adjusting the blade pitch angle $\theta$ or the azimuthal position $\beta$ of the blades (the Voith cycloidal rudder is a two-bladed vertically oriented stern cycloidal propeller used to yaw ships, while also serving as a redundant propulsor). Although, we must keep in mind that reducing the blade count may increase the propeller speed, load, and power (power $\propto$ propeller speed ${ }^{3}$ ) required to produce a target thrust (assuming other propeller and UUV parameters remain unchanged). Such change requires careful consideration in order to avoid excessive stress or deflection, and to avoid cavitation and ventilation (if operating near the free surface). Extensive parametric analysis of cycloidal propeller parameters such as propeller geometry, blade planform, blade profile, blade count and blade pitching kinematics has been carried out in Benedict et al. [2010, 2013a,b] and Benedict et al. [2014] for micro-air-vehicles. A similar analysis in flow conditions of unmanned marine vehicles, with consideration of the UUV profile and propeller configuration, will be beneficial to the control system design and hydrodynamic design optimization of marine cycloidal propellers. However, such an analysis was outside the scope of the present investigation.

\section{Dr. Dana R. Yoerger}

Dept of Applied Ocean Physics and Engineering, Woods Hole Oceanographic Institution - Massachusetts, USA

This paper describes important work that can enable substantial improvements in performance of UUVs in tasks that require maneuverability and conventional transit. Cycloidal propellers are well-established for large vessels (particularly tugs and ferries) but are not available for small vehicles where their versatility would be extremely useful. This approach to UUV maneuvering is refreshing (in my opinion) albeit less "fashionable" than 
biomimetic approaches. As cycloids are powered by a conventional rotary actuator, they enjoy a huge advantage over schemes that involve flapping, which usually require linear actuators.

The analysis is very thorough, although I'm a bit unclear about the importance of the dynamics of the propeller blades (mentioned in the text near equation 7). Does this account for all of the dynamic effect as the blade experiences time-varying flow? This seems particularly important for a cycloidal drive as the advance velocity and angle-of-attack are constantly changing. I've always understood, informally, for a change in flow or angle of attack that lift and drag become fully developed when the blade (or wing) has moved a fraction of a chord length (maybe 1/3). So the dynamic response depends on the speed. A clarification of this point would be useful.

The authors point out that their future work will consider efficiency. This is a key point and will be a very important factor in determining the suitability of these drives for specific application. While beyond the scope of this investigation, mechanical reliability will be another key factor.

The experimental results are impressive and exciting. They form a solid basis for future experiments where these actuators are used in actual closed-loop vehicle tests.

The authors might mention that a version of a cycloidal drive is available for model boats, although it is not well-suited to submerged vehicles as sold:

https: / / www. cornwallmodelboats.co.uk/

acatalog/Voith_Schneider_Drives.html

Minor typos: In the abstract, "it's" should be "its" about half-way through

In summary, this is exciting, important work backed up by solid analysis and experiment. I look forward to seeing the next steps as they unfold.

Authors' response - Thank you for the positive comments. You rightly mention that for a change in angle of attack of a blade, the lift and drag become fully developed when the blade (or wing) has moved a fraction of the chord length. This is because a trailing vortex is generated as soon as a change in angle of attack of the blade occurs, and it takes some time to shed this trailing vortex. However, in a cycloidal propeller blade, the pitch and angle of attack change continuously. As a result, the blade continuously sheds trailing vortices which create an unsteady dynamic effect and prevent the lift and drag from becoming fully developed. This is captured using a modified Theodorsen's approach (page 6-7, Eq. (7), in the revised paper). Theodorsen's function $C\left(k_{f}\right)$ is a function of reduced frequency $k_{f}$. This reduced frequency contains information regarding how fast the blade is oscillating compared to the incoming flow speed.

Moreover, the rapid pitching of a cycloidal propeller blade on a curvilinear path has additional unsteady dynamic effects on the blade such as formation of leading-edge vortices, a nonlinear dynamic virtual camber and a non-uniform dynamic inflow. These effects have been rigorously modeled in Halder et al. [2018] and are incorporated in the present work.

We also agree with the critical points about reliability and efficiency. Cycloidal propellers are more complex than conventional propellers, and hence it is important to carefully consider the material and mechanical connections to ensure reliable operations. One of the disadvantage of cycloidal propellers is its reduced efficiency at higher speeds, and hence future work includes optimization of the propellers and UUV based on the targeted mission profile to enhance overall efficiency, maneuverability and reliability. 